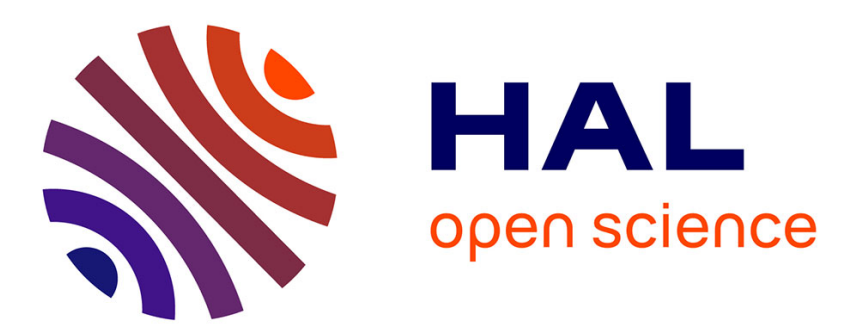

\title{
A versatile interface model for thermal conduction phenomena and its numerical implementation by XFEM
}

Jt Liu, St Gu, Eric Monteiro, Q.C. He

\section{To cite this version:}

Jt Liu, St Gu, Eric Monteiro, Q.C. He. A versatile interface model for thermal conduction phenomena and its numerical implementation by XFEM. Computational Mechanics, 2014, 53, pp.825-843. 10.1007/s00466-013-0933-9 . hal-01174678

\section{HAL Id: hal-01174678 \\ https://hal.science/hal-01174678}

Submitted on 1 Jun 2017

HAL is a multi-disciplinary open access archive for the deposit and dissemination of scientific research documents, whether they are published or not. The documents may come from teaching and research institutions in France or abroad, or from public or private research centers.
L'archive ouverte pluridisciplinaire HAL, est destinée au dépôt et à la diffusion de documents scientifiques de niveau recherche, publiés ou non, émanant des établissements d'enseignement et de recherche français ou étrangers, des laboratoires publics ou privés. 


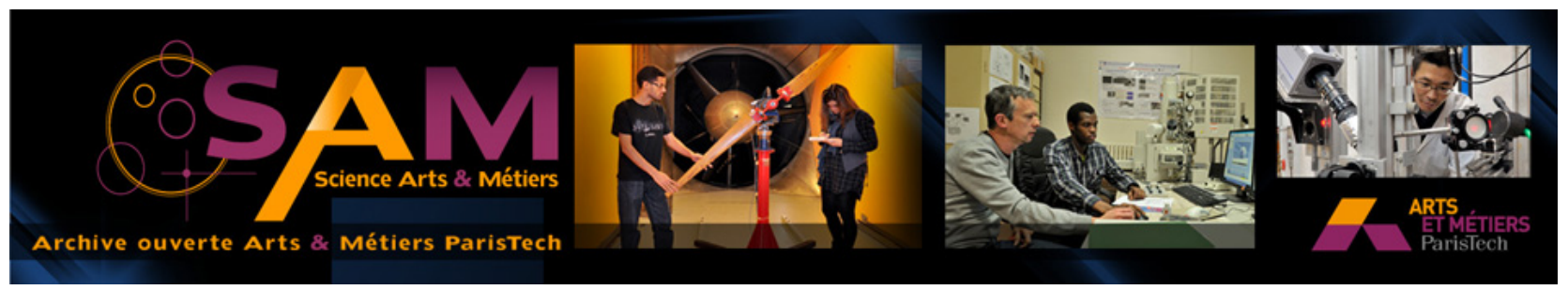

Science Arts \& Métiers (SAM)

is an open access repository that collects the work of Arts et Métiers ParisTech researchers and makes it freely available over the web where possible.

This is an author-deposited version published in: http://sam.ensam.eu

Handle ID: .http://hdl.handle.net/10985/9750

\section{To cite this version :}

JT LIU, ST GU, Eric MONTEIRO - A versatile interface model for thermal conduction phenomena and its numerical implementation by XFEM - Computational Mechanics - Vol. 53, p.825-843 2014 


\title{
A versatile interface model for thermal conduction phenomena and its numerical implementation by XFEM
}

\author{
J. T. Liu - S. T. Gu · E. Monteiro - Q. C. He
}

\begin{abstract}
A general interface model is presented for thermal conduction and characterized by two jump relations. The first one expresses that the temperature jump across an interface is proportional to the interfacial average of the normal heat flux while the second one states that the normal heat flux jump is proportional to the surface Laplacian of the interfacial average of the temperature. By varying the two scalar proportionality parameters, not only the Kapitza resistance and highly conducting interface models can be retrieved but also all the intermediate cases can be covered. The general interface model is numerically implemented by constructing its weak form and by using the level-set method and XFEM. The resulting numerical procedure, whose accuracy and robustness are thoroughly tested and discussed with the help of a benchmark problem, is shown to be efficient for solving the problem of thermal conduction in particulate composites with various imperfect interfaces.
\end{abstract}

Keywords Interface - Interphase - Discontinuity $\cdot$ XFEM . Level set

J.T. Liu · S.T. Gu $(\bowtie) \cdot$ Q. C. He $(\bowtie)$

School of Mechanical Engineering, Southwest Jiaotong University, Chengdu 610031, China

e-mail: gust@home.swjtu.edu.cn

Q. C. He

e-mail: qi-chang.he@univ-paris-est.fr

E. Monteiro · Q. C. He

Laboratoire Modélisation et Simulation Multi Echelle,

MSME UMR 8208 CNRS, Université Paris-Est,

5 Bd Descartes, 77454 Marne-la-Vallée Cedex 2, France

\section{Introduction}

The interface between two phases of a material, or between two elements of a structure, is classically assumed to be perfect. In the context of thermal conduction, an interface is said to be perfect if the requirement that both the temperature and the normal heat flux are continuous across it is satisfied. In many practical situations and for divers reasons, this requirement fails to be fulfilled, so that imperfect interfaces have to be considered. There are two well-known and widely used models to describe thermal imperfect interfaces. The first one is Kapitza's thermal resistance or lowly conducting (LC) interface model [1-7]. According to this model, the temperature is discontinuous and presents a jump across an interface while the normal heat flux is continuous and proportional to the corresponding temperature jump across the interface. The second one is the highly conducting (HC) interface model [8-14]. In contrast to the former, the latter stipulates that the temperature is continuous across an interface whereas the normal heat flux exhibits a jump across it, which is proportional to the surface Laplacian of the temperature. From the standpoint of numerical analysis, it is important to note that the LC interface model involves a strong discontinuity and the $\mathrm{HC}$ one comprises a weak discontinuity.

The extended finite element method (XFEM), which was initially proposed in the works $[15,16]$ and has since then been considerably developed, is particularly suitable and efficient for the numerical treatment of interfacial discontinuities [12,17-26]. Yvonnet et al. [20] first applied XFEM and the level-set method (LSM) to numerically implement the elastic membrane interface model, which can be viewed as the elastic counterpart of the $\mathrm{HC}$ interface model, and to compute the size-dependent effective elastic moduli of nanocomposites. After that, Yvonnet et al. [5,12] and Zhu et al. [25] further developed the XFEM/LSM approach for the numerical 
implementation of the $\mathrm{HC}$ and LC interface models and the elastic spring-layer interface model which can be regarded as the elastic counterpart of the LC interface model.

The LC and HC interface models were initially proposed on the basis of some phenomenological observations. By considering a physically sound configuration where a thin interphase of uniform weak thickness is perfectly bonded to two neighboring bulk phases, Sanchez Palencia [27] and Pham Huy and Sanchez Palencia [28] applied mathematical asymptotic analysis to show that, when the thickness of the interphase tends to zero, the resulting imperfect interface model corresponds to: (i) Kapitza's thermal resistance model if the conductivity of the interphase is much lower than the ones of the surrounding bulk phases; (ii) the $\mathrm{HC}$ interface model if the inverse takes place. Thus, the LC and HC interface models are associated to two opposite extreme situations. Later, Hashin [29] and Benveniste [30] considered the same physical configuration as in Sanchez Palencia [27] and Pham Huy and Sanchez Palencia [28] and applied Taylor's expansion to obtain a more general thermal imperfect interface model in which the temperature and normal heat flux can be simultaneously discontinuous across an interface. This general imperfect interface model includes the LC and HC interface models as particular cases, because it allows retrieving them upon requiring the contrast between the conductivity of the interphase and those of the neighboring phases to be sharp. In a recent work, Gu and He [31] derived a general imperfect interface model for coupled linear multifield phenomena and expressed the corresponding interfacial jump relations in a coordinate-free and compact manner.

To the best of the authors' knowledge, the aforementioned general imperfect interface model involving both the temperature and normal heat flux jumps for thermal conduction has never been numerically implemented within the XFEM/LSM framework. This observation has motivated the present work aiming to fill this gap. Compared with the LC and $\mathrm{HC}$ interface models, the general interface model is much more complicated to be numerically implemented for two reasons. First, the governing equations of the model consists of a firstorder partial differential equation (PDE) and a second-order PDE defined on a curved surface. Next, a weak discontinuity and a strong discontinuity occur simultaneously.

The present work concerns the general interface model derived for the most important case from a practical point of view, i.e. the case where the interphase and the surrounding bulk phase are all isotropic. In the previous relevant studies $[30,31]$, the jump relations characterizing the general model in this case are specified by the equations involving 4 independent parameters. However, in the recent paper [32] , we have shown for the first time that those equations are in fact equivalent to Eqs. (3) and (4) which are simpler and contain only two independent parameters. Further, Eqs. (3) and (4) lend them to interpretation. Precisely, Eq. (3) means that the interfacial jump of the temperature is proportional to the interfacial average of the normal heat flux via a thermal resistance compensating parameter; Eq. (4) signifies that the interfacial jump of the normal heat flux is proportional to the surface Laplacian of the interfacial average of the temperature through a thermal conductivity compensating parameter. According as the thermal conductivity or resistance compensating parameter is set to zero, we recover the $\mathrm{LC}$ or $\mathrm{HC}$ interface model. When both of them are different from zero, the general model describes the intermediate situations between the two opposite extreme cases characterized by the LC and HC models. If both of them are equal to zero, we have the perfect interface. So, the general interface model considered in this work is simple and versatile in studying thermal interfacial effects.

Most of the works in which XFEM and LSM are used to numerically treat interfacial discontinuities are concerned either with weak or strong discontinuity, as mentioned above. Those accounting for both the weak and strong interfacial discontinuities are quite limited. Dolbow and Harari $[33,34]$ proposed a finite element method based on the Nitsche technique to deal with a transport problem in which weak and strong interfacial discontinuities intervene. To study surface and interface effects on the elastic properties of nanomaterials, Farsad et al. [23] elaborated an XFEM/LSM approach similar to the one of Yvonnet et al. [20] but with the capacity to handle strain and displacement discontinuities simultaneously. In the present work, an XFEM/LSM procedure is further developed to numerically implement the general imperfect interface model for steady thermal conduction.

The paper is organized as follows. In the next section, the physical background of the general interface model for steady thermal conduction is recalled, and the equations characterizing it with isotropic interphase and bulk phases are recasted so as to take a considerably simplified but equivalent form with only two parameters. In Sect. 3, the strong and weak formulations are given for the boundary value problem of steady thermal conduction in a particulate composite with the interfaces characterized by the general interface model. In particular, by varying the parameters of the interface model, the weak formulations for LC and HC interfaces can be easily retrieved. Section 4 is dedicated to the discretization of the weak formulation by XFEM and LSM. Since the temperature and its gradient exhibit jumps across an interface, two kinds of enrichment functions are used in the finite element approximation of the temperature field. In Sect. 5, a benchmark problem is first analytically and exactly solved. An convergence analysis is then carried out for the numerical procedure proposed in Sect. 4 with the aid of the analytical exact solution for the benchmark problem. The efficiency and robustness of the proposed numerical procedure are tested for extreme and intermediate cases. The effects of the shapes and 
distribution of the inclusions on the temperature field are also examined. In Sect. 6, a few concluding remarks are drawn.

\section{A simple and versatile interface model for thermal conduction}

We are interested in the phenomenon of thermal conduction in a composite consisting of a matrix in which particulates are embedded. The interface between the matrix and a generic particulate is assumed to be imperfect. A physically sound and mathematically rigorous way to obtain a class of imperfect interface models $[30,31,35]$ is now recalled as follows.

We first consider a three-phase configuration where a particulate is bonded to the matrix through an interphase of small uniform thickness $h$ (Fig. 1a). In this threephase configuration, the domains occupied by the matrix, a generic particulate $i$ and their interphase are denoted by $\hat{\Omega}^{(M)}, \hat{\Omega}^{(i)}$ and $\Omega^{(0)}$. The interface $S_{M}$ between $\hat{\Omega}^{(M)}$ and $\Omega^{(0)}$, and the interface $S_{i}$ between $\hat{\Omega}^{(i)}$ and $\Omega^{(0)}$, are both taken to be perfect. The middle surface of the interphase $\Omega^{(0)}$ is symbolized by $\Gamma_{i}$. The unit vector normal to $\Gamma_{i}$ (also to $S_{M}$ and $S_{i}$ ) oriented from $\hat{\Omega}^{(i)}$ to $\hat{\Omega}^{(M)}$ is noted as $\mathbf{n}_{i}$. The constituent materials of $\hat{\Omega}^{(M)}, \hat{\Omega}^{(i)}$ and $\Omega^{(0)}$ are assumed to be individually homogeneous and comply with the Fourier law of thermal conduction, so that

$\mathbf{q}^{(r)}=-\mathbf{K}^{(r)} \nabla \varphi^{(r)} \quad(r=M, i, 0)$

where $\varphi^{(r)}$ and $\mathbf{q}^{(r)}$ represent the temperature and heat flux fields defined over $\hat{\Omega}^{(r)}$, respectively, and $\mathbf{K}^{(r)}$ is the secondorder thermal conductivity tensor of the material forming $\hat{\Omega}^{(r)}$, which is positive definite.

Next, a two-phase configuration is obtained through replacing the interphase in the aforementioned three-phase configuration by an imperfect interface of null thickness whose geometrical location is that of the middle surface $\Gamma_{i}$ (Fig. 1b). At the same time, the materials forming $\hat{\Omega}^{(M)}$ and $\hat{\Omega}^{(i)}$ are extended to $\Gamma_{i}$, respectively, so that $\hat{\Omega}^{(M)}$ and $\hat{\Omega}^{(i)}$ are replaced by $\Omega^{(M)}$ and $\Omega^{(i)}$ separated

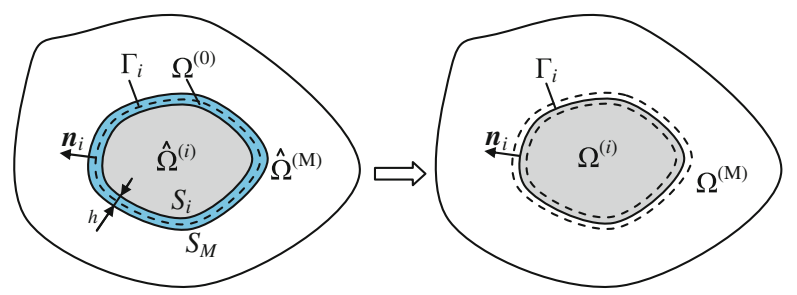

(a)

(b)

Fig. 1 Physical configurations used to derive the general thermal imperfect interface model: a three-phase configuration; b two-phase configuration directly by $\Gamma_{i}$ in the two-phase configuration. The interfacial relations to be satisfied by the imperfect interface $\Gamma_{i}$ are required to be such that the two-phase configuration is, to within a fixed error, equivalent to the initial three-phase configuration. More precisely, the jumps of the temperature and normal heat flux across the zone bounded by the surfaces $S_{M}$ and $S_{i}$ in two-phase configuration are required to be equal, to within a given error, to those across the interphase $\Omega^{(0)}$ in the three-phase configuration.

In Refs. [30,31] and [35], the general interfacial relations to be satisfied by $\Gamma_{i}$ in the two-phase configuration were derived. These interfacial relations present several salient features: (i) both the temperature and normal heat flux are allowed to be discontinuous across the imperfect interface $\Gamma_{i}$; (ii) the contrast between the thermal conductivities of the matrix, particulate and interphase can be arbitrary; (iii) each of $\mathbf{K}^{(M)}$, $\mathbf{K}^{(i)}$ and $\mathbf{K}^{(0)}$ can be anisotropic; (iv) each of $\mathbf{K}^{(M)}, \mathbf{K}^{(i)}$ and $\mathbf{K}^{(0)}$ can be inhomogeneous. In what follows, we are interested in the most important particular case when each of $\mathbf{K}^{(M)}, \mathbf{K}^{(i)}$ and $\mathbf{K}^{(0)}$ is homogeneous and isotropic, so that

$\mathbf{K}^{(r)}=k^{(r)} \mathbf{I} \quad(r=M, i, 0)$

where $k^{(r)}$ is a positive constant and $\mathbf{I}$ is the second-order identity tensor. In this situation, Gu et al. [32] have recently proved that the general interfacial relations can be recast into

$$
\begin{aligned}
{[[\varphi]]_{i} } & =\frac{h}{2} a^{(i)}\left\langle q_{n}\right\rangle_{i}+0\left(h^{2}\right), \\
{\left[\left[q_{n}\right]\right]_{i} } & =\frac{h}{2} b^{(i)} \Delta_{s}\langle\varphi\rangle_{i}+0\left(h^{2}\right) .
\end{aligned}
$$

In these expressions, $q_{n}$ denotes the normal heat component given by $q_{n}=\mathbf{q} \cdot \mathbf{n}^{(i)} ;[[\bullet]]_{i}$ and $\langle\bullet\rangle_{i}$ are the interfacial jump and average operators relative to $\Gamma_{i}$, which are defined by

$[[\bullet]]_{i}=\bullet^{(+)}-\bullet^{(-)}, \quad\langle\bullet\rangle_{i}=\frac{1}{2}\left(\bullet^{(+)}+\bullet^{(-)}\right)$.

where $\bullet^{(+)}$and $\bullet^{(-)}$stand for the values of a quantity $\bullet$ evaluated at $\Gamma_{i}$ on the matrix side and the $i$ th inclusion side, respectively. In addition, the surface gradient $\nabla_{s}(\bullet)$ and Laplacian $\Delta_{S}(\bullet)$ of a scalar quantity $(\bullet)$ are, by definition, given by

$$
\begin{aligned}
& \nabla_{s}(\bullet)=\nabla(\bullet) \cdot \mathbf{T}^{(i)}, \\
& \Delta_{S}(\bullet)=\operatorname{div}_{S}\left(\nabla_{s}(\bullet)\right)=\nabla\left(\nabla_{s}(\bullet)\right): \mathbf{T}^{(i)} .
\end{aligned}
$$

where $\mathbf{T}^{(i)}=\mathbf{I}-\mathbf{n}^{(i)} \otimes \mathbf{n}^{(i)}$ is the tangential projection operator. The material parameters $a^{(i)}$ and $b^{(i)}$ in (3) and (4) depend on the material parameters $k^{(M)}, k^{(i)}$ and $k^{(0)}$ as follows:

$a^{(i)}=\frac{1}{k^{(M)}}+\frac{1}{k^{(i)}}-\frac{2}{k^{(0)}}, b^{(i)}=2 k^{(0)}-k^{(i)}-k^{(M)}$. 
The general isotropic thermal imperfect interface model characterized by (3) and (4) together with (7) will be numerically implemented in the afterward sections. The relation (3) means that the interfacial jump of the temperature is proportional to the interfacial average of the normal heat flux while the expression (4) signifies that the interfacial jump of the normal heat flux is proportional to the surface Laplacian of the interfacial average of the temperature. Both the material parameters $a^{(i)}$ and $b^{(i)}$ involved in the model can be positive or negative. From the physical point of view, $a^{(i)}$ and $b^{(i)}$ can be, respectively, interpreted as thermal resistance and conductivity compensating parameters in the process of replacing the interphase in the three-phase configuration (Fig. 1a) by an imperfect interface $\Gamma_{i}$ in the two-phase configuration (Fig. 1b) with the inclusion and matrix phases extended up to $\Gamma_{i}$.

From Eqs. (3), (4) and (7), we can retrieve the well-known Kapitza's thermal resistance interface model [5] and the HC interface model [12] as two extreme particular cases. First, when $h$ is very small and when $k^{(0)} \ll k^{(M)}$ and $k^{(0)} \ll$ $k^{(i)}$, we have $h a^{(i)} \approx-2 h / k^{(0)}$ and $h b^{(i)} \approx 0$ to within an error of order $0(h)$. In this case, we obtain Kapitza's thermal resistance interface model:

$[[\varphi]]_{i}=-\frac{h}{k^{(0)}}\left\langle q_{n}\right\rangle_{i}+0(h)$,

$\left[\left[q_{n}\right]\right]_{i}=0(h)$.

Second, when $h$ is very small and when $k^{(0)} \gg k^{(M)}$ and $k^{(0)} \gg k^{(i)}$, we can deduce from Eqs. (3), (4) and (7) that $h a^{(i)} \approx 0, h b^{(i)} \approx 2 h k^{(0)}$ and

$[[\varphi]]_{i}=0(h)$,

$\left[\left[q_{n}\right]\right]_{i}=h k^{(0)} \Delta_{s}\langle\varphi\rangle_{i}+0(h)$.

These two relations characterize the $\mathrm{HC}$ imperfect interface model.

It is interesting to mention two very special situations. The first corresponds to the case where $2 k^{(0)}=k^{(i)}+k^{(M)}$. Then, it follows from (3), (4) and (7) that

$[[\varphi]]_{i}=-\frac{h a^{(i)}}{2}\left\langle q_{n}\right\rangle_{i}+0\left(h^{2}\right)$,

$\left[\left[q_{n}\right]\right]_{i}=0\left(h^{2}\right)$.

The second takes place when $1 / k^{(M)}+1 / k^{(i)}=2 / k^{(0)}$. In such a situation, it is immediate from (3), (4) and (7) that

$[[\varphi]]_{i}=0\left(h^{2}\right)$,

$\left[\left[q_{n}\right]\right]_{i}=\frac{h b^{(i)}}{2} \Delta_{s}\langle\varphi\rangle_{i}+0\left(h^{2}\right)$.

Note that, under the aforementioned very special conditions, two pairs of relations (12)-(13) and (14)-(15) are exact to within an error of order $0\left(h^{2}\right)$, in contrast with (8)-(9) and (10)-(11) which are exact to within an error of order $0(h)$.

\section{Thermal conduction in a particulate composite with imperfect interfaces: strong and weak formulations}

The problem under investigation concerns a particulate composite consisting of $m$ homogeneous particulate phases embedded in a homogeneous matrix phase (see Fig. 2). Let $\Omega$ be the 3D domain occupied by the composite and $\partial \Omega$ the external boundary of $\Omega$. The subdomains of $\Omega$ inhabited by the matrix phase and the $j$ th particulate phase are designated by $\Omega^{(M)}$ and $\Omega^{(j)}$ such that

$\Omega=\Omega^{(M)} \cup \Omega^{(1)} \cup \Omega^{(2)} \cup \cdots \cup \Omega^{(m)}$.

The interface between $\Omega^{(M)}$ and $\Omega^{(j)}(j=1,2, \ldots, m)$ is symbolized by $\Gamma_{j}=\Omega^{(M)} \cap \Omega^{(j)}$. The particulates are assumed to be distributed inside the matrix in such a way that any particulate does not intersect the composite boundary $\partial \Omega$ and there is no contact between any two particulates. Thus, the boundary $\partial \Omega^{(j)}$ of the $j$ th particulate and the one of the matrix $\Omega^{(M)}$ can be written as

$\partial \Omega^{(j)}=\Gamma_{j} \quad \partial \Omega^{(M)}=\partial \Omega \cup \Gamma_{1} \cup \Gamma_{2} \cup \cdots \cup \Gamma_{m}$.

In what follows, $\mathbf{n}_{M}$ stands for the outward unit vector normal to $\partial \Omega$ and $\mathbf{n}_{j}$ the unit vector normal to $\Gamma_{j}$ oriented from $\Omega^{(j)}$ into $\Omega^{(M)}$.

The steady heat conduction in the absence of heat source is governed by the energy conservation equation

$\operatorname{div} \mathbf{q}^{(l)}=0 \quad$ in $\Omega^{(l)} \quad(l=M, 1,2, \ldots, m)$

where $\mathbf{q}^{(l)}$ represents the heat flux vector defined over $\Omega^{(l)}$ and is related to the temperature field $\varphi^{(l)}$ through the isotropic Fourier law

$\mathbf{q}^{(l)}=-k^{(l)} \nabla \varphi^{(l)}$

with $k^{(l)}$ the conductivity of phase $\Omega^{(l)}$. The interface $\Gamma_{j}$ between $\Omega^{(M)}$ and $\Omega^{(j)}$ is assumed to be imperfect and described by Eqs. (3) and (4). The boundary conditions prescribed on $\partial \Omega$ are the following ones:

$\left\{\begin{aligned} \varphi & =\bar{\varphi} & & \text { on } \partial \Omega_{\varphi} \\ q_{n} & =\bar{q}_{n} & & \text { on } \partial \Omega_{q} .\end{aligned}\right.$

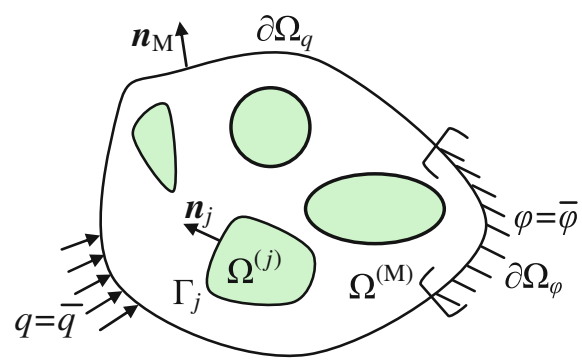

Fig. 2 Schematic of the composite material under investigation 
where $\bar{\varphi}$ and $\bar{q}_{n}$ denote the temperature and normal heat flux imposed on $\partial \Omega_{\varphi}$ and $\partial \Omega_{q}$ with $\partial \Omega=\partial \Omega_{\varphi} \cup \partial \Omega_{q}$ and $\partial \Omega_{\varphi} \cap$ $\partial \Omega_{q}=\varnothing$.

Equations (18)-(20) together with the interfacial relations (3) and (4) constitute the strong formulation for the boundary value problem of steady thermal conduction in the composite $\Omega$. For the numerical solution of this problem, the corresponding weak formulation is needed. Thus, we first introduce a piecewise differentiable virtual temperature field $\delta \varphi$ defined over $\Omega$, such that $\delta \varphi=0$ on $\partial \Omega_{\varphi}$. Then, starting from the energy conservation Eq. (18), we can show that

$$
\begin{aligned}
\int_{\Omega^{(M)}} \mathbf{q}^{(M)} \cdot \nabla \delta \varphi^{(M)} d v & =\int_{\partial \Omega_{q}} q_{n}^{(M)} \delta \varphi^{(M)} d s \\
& -\sum_{j=1}^{m} \int_{\Gamma_{j}} q_{n}^{(j)} \delta \varphi^{(j)} d s, \\
\int_{\Omega^{(j)}} \mathbf{q}^{(j)} \cdot \nabla \delta \varphi^{(j)} d v & =\int_{\Gamma_{j}} q_{n}^{(j)} \delta \varphi^{(j)} d s .
\end{aligned}
$$

Adding these two equations leads to

$$
\int_{\Omega} \mathbf{q} \cdot \nabla(\delta \varphi) d v+\sum_{j=1}^{m} \int_{\Gamma_{j}}\left[\left[q_{n} \delta \varphi\right]\right]_{j} d s=\int_{\partial \Omega_{q}} q_{n} \delta \varphi d s
$$

with

$$
\begin{aligned}
{\left[\left[q_{n} \delta \varphi\right]\right]_{j} } & =q_{n}^{(M)} \delta \varphi^{(M)}-q_{n}^{(j)} \delta \varphi^{(j)} \\
& =q_{n}^{(+)} \delta \varphi^{(+)}-q_{n}^{(-)} \delta \varphi^{(-)} .
\end{aligned}
$$

Using the identity (see, e.g., Gurtin and Murdoch [36])

$\left[\left[q_{n} \delta \varphi\right]\right]_{j}=\left[\left[q_{n}\right]\right]_{j}\langle\delta \varphi\rangle_{j}+\left\langle q_{n}\right\rangle_{j}[[\delta \varphi]]_{j}$,

and accounting for the interfacial relations (3) and (4), we obtain

$\left[\left[q_{n} \delta \varphi\right]\right]_{j}=\frac{h}{2} b^{(j)} \Delta_{s}\langle\varphi\rangle_{j}\langle\delta \varphi\rangle_{j}+\frac{2}{h a^{(j)}}[[\varphi]]_{j}[[\delta \varphi]]_{j}$.

Introducing (19) and (25) into (22), it follows that

$$
\begin{aligned}
& -\int_{\Omega} k \nabla \varphi \cdot \nabla(\delta \varphi) d v+\frac{h}{2} \sum_{j=1}^{m} \int_{\Gamma_{j}} b^{(j)} \Delta_{s}\langle\varphi\rangle_{j}\langle\delta \varphi\rangle_{j} d s \\
& \quad+\frac{2}{h} \sum_{j=1}^{m} \int_{\Gamma_{j}} \frac{1}{a^{(j)}}[[\varphi]]_{j}[[\delta \varphi]]_{j} d s=\int_{\partial \Omega_{q}} q_{n} \delta \varphi d s .
\end{aligned}
$$

By Stokes' divergence theorem, we have

$$
\int_{\Gamma_{j}} b^{(j)} \Delta_{s}\langle\varphi\rangle_{j}\langle\delta \varphi\rangle_{j} d s=-\int_{\Gamma^{(j)}} b^{(j)} \nabla_{s}\langle\varphi\rangle_{j} \cdot \nabla_{s}\langle\delta \varphi\rangle_{j} d s
$$

while the interface $\Gamma^{(j)}$ is taken to be closed. Finally, the weak formulation of the problem in question is given by

$$
\begin{aligned}
& \int_{\Omega} k \nabla \varphi \cdot \nabla(\delta \varphi) d v+\frac{h}{2} \sum_{j=1}^{m} \int_{\Gamma_{j}} b^{(j)} \nabla_{s}\langle\varphi\rangle_{j} \cdot \nabla_{s}\langle\delta \varphi\rangle_{j} d s \\
& -\frac{2}{h} \sum_{j=1}^{m} \int_{\Gamma_{j}} \frac{1}{a^{(j)}}[[\varphi]]_{j}[[\delta \varphi]]_{j} d s=-\int_{\partial \Omega_{q}} q_{n} \delta \varphi d s .
\end{aligned}
$$

The weak formulation (28) is more compact and more structured than the one provided by $\mathrm{Gu}$ et al. [35]. Moreover, it includes as special cases the weak formulations established by Yvonnet et al. [5, 12] for the HC imperfect interface model and for Kapitza's resistance imperfect interface model. Indeed, by setting $h b^{(j)}=2 h k^{(0)}$ and by using $h a^{(j)}=0$ as a penalty parameter in (28), we have

$$
\begin{aligned}
& \int_{\Omega} k \nabla \varphi \cdot \nabla(\delta \varphi) d v+h \sum_{j=1}^{m} \int_{\Gamma^{(j)}} k^{(0)} \nabla_{s}\langle\varphi\rangle_{j} \cdot \nabla_{s}\langle\delta \varphi\rangle_{j} d s \\
& \quad=-\int_{\partial \Omega_{q}} q_{n} \delta \varphi d s
\end{aligned}
$$

by putting $h a^{(j)}=-2 h / k^{(0)}$ and $h b^{(j)}=0$ in Eq. (28), it comes that

$$
\begin{aligned}
& \int_{\Omega} k \nabla \varphi \cdot \nabla(\delta \varphi) d v+\frac{1}{h} \sum_{j=1}^{m} \int_{\Gamma_{j}} k^{(0)}[[\delta \varphi]]_{j}[[\delta \varphi]]_{j} d s \\
& =-\int_{\partial \Omega_{q}} q_{n} \delta \varphi d s .
\end{aligned}
$$

The weak forms Eqs. (29) and (30) correspond to those provided and numerically implemented by Yvonnet et al. [5] and [12].

\section{Discretization by XFEM and LSM}

Now, we turn to the elaboration of a numerical strategy to solve the boundary value problem whose weak formulation is given by expression (28). In this problem, both the strong and weak interfacial discontinuities, namely the discontinuity of the temperature and that of its gradient, are involved. To deal with these discontinuities numerically, we adopt the XFEM and the LSM in what follows.

Recall that the basic idea of XFEM applied to the problem under consideration consists in adding appropriate local enrichment functions to the standard finite element approximation so as to account for interfacial jumps. More precisely, considering a domain $\Omega$ containing imperfect interfaces $\Gamma_{j}$ and discretized by $n_{n d}$ nodes that do not necessarily match 
$\Gamma_{j}$, the temperature field over $\Omega$ can be approximated by (see, e.g., [23])

$$
\begin{aligned}
\varphi^{h}(\mathbf{x})= & \sum_{s=1}^{n_{n d}} N_{s}(\mathbf{x}) \varphi_{s}+\sum_{p=1}^{m_{n d}} N_{p}(\mathbf{x}) \chi_{p}(\mathbf{x}) \bar{\varphi}_{p} \\
& +\sum_{p=1}^{m_{n d}} N_{p}(\mathbf{x}) \psi_{p}(\mathbf{x}) \overline{\bar{\varphi}}_{p} .
\end{aligned}
$$

In this expression, $N_{s}$ are standard shape functions; $\varphi_{s}$ denotes the temperature value at the $s$ th node; the unknowns $\bar{\varphi}_{p}$ and $\overline{\bar{\varphi}}_{p}$ are the enriched degrees of freedom (DOFs) associated with the strong and weak discontinuities; the enrichment functions $\chi_{p}$ and $\psi_{p}$ are defined for the nodes belonging to the elements cut by an imperfect interface; the number $m_{n d}$ of enriched nodes is required to be $m_{n d} \leq n_{n d}$. In what follows, the four-node tetrahedral linear finite element is employed. Concerning the use of higher-order finite elements, we refer to [37].

A key step towards solving the problem in question lies in selecting appropriate enrichment functions. These functions are required to fulfill two tasks: (i) to locate the position of an imperfect interface inside the intercepted elements; (ii) to describe the discontinuities of the temperature and the normal heat flux across the imperfect interface. For the achievement of the task (i) and with no loss of generality, a generic imperfect interface $\Gamma$ is defined as the zero level-set of a scalar-value function $\phi$ :

$\Gamma=\left\{\mathbf{x} \in \mathbb{R}^{3} \mid \phi(\mathbf{x})=0\right\}$.

Thus, the function $\phi$ takes negative values on one side of the interface and positive ones on its other side. To be consistent with the previous formulation of the problem, we assume that the level-set function $\phi$ is defined so as to be positive in the matrix phase and negative in any inclusion phase. In the context of finite elements, the level-set function is approximated by

$\phi^{h}(\mathbf{x})=\sum_{s=1}^{n_{e}} N_{s}(\mathbf{x}) \phi_{s}$

where $\phi_{s}$ denotes the value of $\phi$ at the $s$ th node and $n_{e}$ is the total number of nodes in an element. In addition, the unit normal vector at point $\mathbf{x}$ on $\Gamma$ can be numerically evaluated by

$\mathbf{n}^{h}(\mathbf{x})=\frac{\nabla \phi^{h}(\mathbf{x})}{\left\|\nabla \phi^{h}(\mathbf{x})\right\|} \quad$ with $\quad \nabla \phi^{h}(\mathbf{x})=\sum_{s=1}^{n_{e}} \frac{\partial N_{s}(\mathbf{x})}{\partial \mathbf{x}} \phi_{s}$.

With the help of the level-set function $\phi$ and its nodal values $\phi_{p}$, the following enrichment functions are adopted to describe the temperature and normal heat flux jumps: $\chi_{p}(\mathbf{x})=\operatorname{sign}(\phi(\mathbf{x}))-\operatorname{sign}\left(\phi_{p}\right)$,

$\psi_{p}(\mathbf{x})=\sum_{s=1}^{n_{e}} N_{s}(\mathbf{x})\left|\phi_{s}\right|-\left|\sum_{s=1}^{n_{e}} N_{S}(\mathbf{x}) \phi_{s}\right|$,

where the function $\operatorname{sign}(\bullet)$ is defined by $\operatorname{sign}(\bullet)=$ $-1,0$ or 1 according as $(\bullet)$ is negative, equal to zero or positive. The derivation of $\chi_{p}$ and $\psi_{p}$ gives

$$
\begin{aligned}
& \frac{\partial \chi_{p}(\mathbf{x})}{\partial \mathbf{x}}=0 \\
& \frac{\partial \psi_{p}(\mathbf{x})}{\partial \mathbf{x}}=\sum_{s=1}^{n_{e}}\left\{\frac { \partial N _ { s } ( \mathbf { x } ) } { \partial \mathbf { x } } \left[\left|\phi_{s}\right|-\operatorname{sign}\left(\sum_{s=1}^{n_{e}} N_{S}(\mathbf{x}) \phi_{S}\right.\right.\right.
\end{aligned}
$$

Formulae (37) and (38) are meaningful provided $\phi(\mathbf{x}) \neq$ 0 and $\sum_{s=1}^{n_{e}} N_{s}(\mathbf{x}) \phi_{s} \neq 0$, respectively.

Equation (35) allows to capture a jump in the temperature field (strong discontinuity) and the shifting procedure guarantees that any nodal value $\varphi_{s}$ keeps its meaning of true temperature. Equation (36) makes it possible to produce a jump in the heat flux field (weak discontinuity) across the imperfect interface since it is related to the temperature gradient. Hence, the use of the enrichment functions (35) and (36) allows accounting for both the jumps within the framework of finite elements. The effect of the enrichment functions on the standard linear finite element shape functions is depicted in Fig. 3 for the 1D case. In addition, as the enrichment function $\psi_{p}$ defined by (36) and its derivative given by (38) are zeros in both the blending elements of type 1 and type 2 [25], no wanted higher order terms [38] need being added in the blending elements. Consequently, additional treatments are not necessary for these elements. Further, the enrichment function $\chi_{p}(\mathbf{x})$ is constant in all blending elements and the partition of unity function $N_{p}(\mathbf{x})$ is of the same order compared with the standard shape function $N_{s}(\mathbf{x})$, so that the unwanted term in the blending elements due to $\chi_{p}(\mathbf{x})$ can be compensated by the standard finite element shape functions [39] and no supplementary treatment is required for the enrichment functions. It is worth noting that the blending elements of type 1 [25] necessitate additional treatment on the DOFs $\bar{\varphi}$. Since $\chi_{p}(\mathbf{x})$ equals to $\operatorname{sign}(\phi(\mathbf{x}))$ on the Gauss integration points when $\mathbf{x}_{p}$ is located on the interface, the thermal conductivity contributed by these elements must be integrated and complementary implement becomes indispensable for such partially enriched elements.

An important aspect to be emphasized is the numerical integration within an intercepted element when discontinuities occur. To ensure accuracy, the intercepted element is subdivided into sub-elements whose edges conform to the interface, so that Gauss quadrature can be used in these 
Fig. 3 Illustration of the enrichments used to capture the in the $1 \mathrm{D}$ case. a Enrichment function $\chi_{p}(x)$. b Enrichment function $\psi_{p}(x)$. c Derivative $\frac{\partial \psi_{p}(x)}{\partial x}$ strong and weak discontinuities

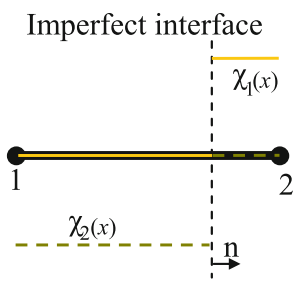

(a)

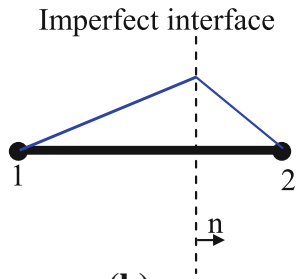

(b)

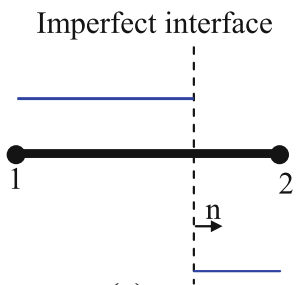

(c)

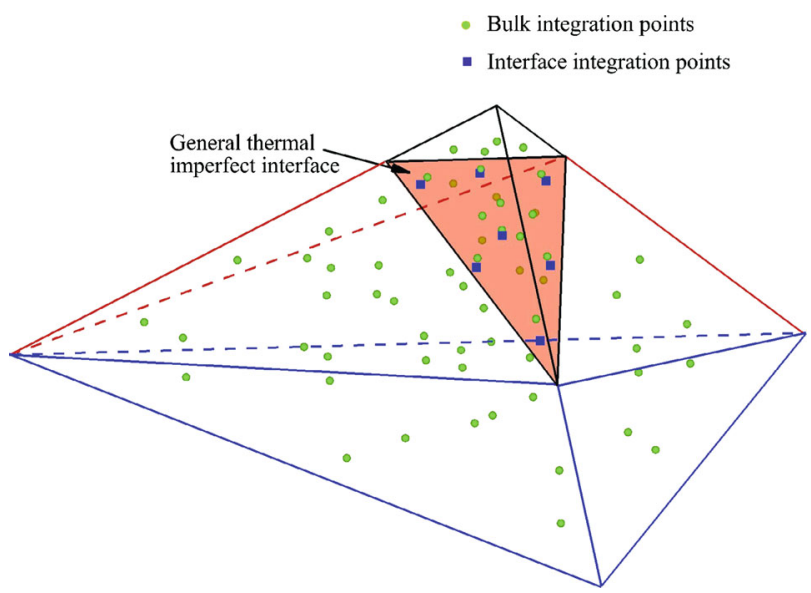

Fig. 4 Illustration of the subdivision of an intercepted element

sub-elements. Furthermore, the usual Gauss rule involving integration points on the interface is employed to evaluate the surface contribution. Figure 4 shows an instance of the subdivision and the distribution of the integration points.

For the aforementioned specific integrands, some quantities must be evaluated on both sides of an imperfect interface to incorporate the interface contribution. The enrichment functions on the two sides of the imperfect interface are taken to be

$$
\begin{aligned}
& \chi_{p}^{(+)}=1-\operatorname{sign}\left(\phi_{p}\right), \chi_{p}^{(-)}=-1-\operatorname{sign}\left(\phi_{p}\right) . \\
& \frac{\partial \psi_{p}^{(+)}(\mathbf{x})}{\partial \mathbf{x}}=\sum_{s=1}^{n_{e}}\left\{\frac{\partial N_{s}(\mathbf{x})}{\partial \mathbf{x}}\left[\left|\phi_{s}\right|-\phi_{s}\right]\right. \\
& \frac{\partial \psi_{p}^{(-)}(\mathbf{x})}{\partial \mathbf{x}}=\sum_{s=1}^{n_{e}}\left\{\frac{\partial N_{s}(\mathbf{x})}{\partial \mathbf{x}}\left[\left|\phi_{s}\right|+\phi_{s}\right] .\right.
\end{aligned}
$$

Finally, the discretization of the weak form (28) yields the following linear discrete system

$$
\left(\mathbf{K}+\mathbf{K}_{s}\right) \Phi=\mathbf{F}
$$

where the vector $\Phi$ contains all the DOFs, i.e. the classical DOFs $\varphi_{s}$ and the enriched DOFs $\bar{\varphi}_{p}$ and $\overline{\bar{\varphi}}_{p}$. The bulk conductivity matrix $\mathbf{K}$ is computed by

$\mathbf{K}=\sum_{s=1}^{n_{e m}} \int_{\Omega_{s}^{e}} \mathbf{B}^{T} \mathbf{K}_{s}^{(l)} \mathbf{B} d \Omega_{s}^{e}$

where $n_{e m}$ is the total number of elements in $\Omega, \Omega_{s}^{e}$ denotes the domain occupied by the $s$ th element, $\mathbf{K}_{s}^{(l)}=$ $k^{(l)} \operatorname{diag}(1,1,1)$ represents the conductivity matrix of the $l$ th phase, the matrix $\mathbf{B}$ is defined through $\nabla \varphi=\mathbf{B} \varphi^{e}$ with $\varphi^{e}$ being the nodal unknowns of an element. The interface conductivity matrix $\mathbf{K}_{s}$ in (41) is provided by

$$
\left.\mathbf{K}_{s}=\sum_{j=1}^{m} \sum_{j^{\prime}=1}^{n_{s}} \int_{\Gamma_{j-j^{\prime}}^{e}} \frac{h}{2} b^{(j)} \widetilde{\mathbf{B}}_{j^{\prime}}^{T} \mathbf{T}^{\left(j^{\prime}\right)} \widetilde{\mathbf{B}}_{j^{\prime}}-\frac{2}{h a^{(j)}} \widetilde{\mathbf{N}}_{j^{\prime}}^{T} \widetilde{\mathbf{N}}_{j^{\prime}}\right] d S^{e}
$$

where $n_{s}$ is the total number of the segments discretizing the $j$ th imperfect surface, $\Gamma_{j_{-} j^{\prime}}^{e}$ stands for the $j /$ th surface segment of the $j$ th imperfect surface, the matrices $\widetilde{\mathbf{B}}_{j^{\prime}}$ and $\widetilde{\mathbf{N}}_{j^{\prime}}$ are defined through $\nabla\langle\varphi\rangle=\widetilde{\mathbf{B}}_{j^{\prime}} \varphi^{e}$ and $[[\varphi]]=\widetilde{\mathbf{N}}_{j^{\prime}} \varphi^{e}$, respectively. The heat flux vector $\mathbf{F}$ in (41) is calculated by

$\mathbf{F}=-\int_{\partial \Omega_{q}} \mathbf{N}^{T} \bar{q}_{n} d S$

with $\mathbf{N}$ being the matrix of shape functions.

\section{Examples and discussions}

In this section, we first analytically solve the problem of thermal conduction in an infinite matrix in which a spherical inclusion is embedded via an imperfect interface described by the jump relations (3) and (4). The numerical procedure presented in the last section is then used to deal with the same problem. Its validity and accuracy are tested against the corresponding analytical exact solution taken as a benchmark. Finally, the numerical procedure validated is applied to solve the problem of thermal conduction inside a composite 
consisting of multiple spherical and non-spherical inclusions inside a matrix with imperfect interfaces.

\subsection{Analytical solution to a benchmark problem}

Consider a spherical inclusion of radius $R$ inside an infinite matrix. The thermal conductivity tensors of the materials forming the inclusion and matrix are isotropic and specified by (2). The interface between the inclusion and the matrix is imperfect and characterized by the relations (3) and (4). The origin of the coordinate system is chosen to coincide with the center of the spherical inclusion. Let the matrix be subjected to a remote uniform temperature gradient, i.e.

$\varphi=\bar{\varphi}=-\mathbf{g}_{0} \cdot \mathbf{x}$ with $\quad\|\mathbf{x}\| \rightarrow+\infty$

where $\mathbf{x}$ is the position vector and $\mathbf{g}_{0}$ is a constant vector. We are interested in the resulting steady thermal conduction problem.

The temperature fields over the matrix and inclusion are postulated as

$\varphi^{(r)}=\mathbf{g}_{0} \cdot \mathbf{x}\left(A^{(r)}+\frac{B^{(r)}}{\|\mathbf{x}\|^{3}}\right.$

where $A^{(r)}$ and $B^{(r)}$ are two constants and the superscript $r$ refers to the inclusion when $r=i$ and to the matrix when $r=$ $M$. It is easy to check that the temperature field (46) satisfies the Laplace equation $\Delta \varphi^{(r)}=0$ governing the temperature field inside the inclusion and inside the matrix.

Accounting for the remote boundary condition (45) in (46), we obtain

$A^{(M)}=-1$.

In addition, due to the fact that the temperature at the center of the inclusion must be finite, we deduce

$B^{(i)}=0$.

Thus, the expressions of $\varphi^{(i)}$ and $\varphi^{(M)}$ reduce to

$\varphi^{(i)}=A^{(i)} \mathbf{g}_{0} \cdot \mathbf{x}$

$\varphi^{(M)}=\mathbf{g}_{0} \cdot \mathbf{x}\left(\frac{B^{(M)}}{\|\mathbf{x}\|^{3}}-1\right.$.

Now, introducing (47) and (48) into the jump relations (3) and (4) yields two linear equations with $A^{(i)}$ and $B^{(M)}$ as the unknowns:

$$
\begin{aligned}
& \left.1-\frac{\delta}{4} a^{(i)} k^{(i)}\right] A^{(i)}+\left(\frac{\delta}{2} a^{(i)} k^{(M)}-1\right) \frac{B^{(M)}}{R^{3}} \\
& =-\frac{\delta}{4} a^{(i)} k^{(M)}-1
\end{aligned}
$$

$$
\begin{aligned}
& \left(\frac{\delta}{2} b^{(i)}+k^{(i)}\right) A^{(i)}+\left(\frac{\delta}{2} b^{(i)}+2 k^{(M)}\right) \frac{B^{(M)}}{R^{3}} \\
& \quad=\frac{\delta}{2} b^{(i)}-k^{(M)}
\end{aligned}
$$

with $\delta=h / R$. Solving (49) and (50) delivers

$$
\begin{aligned}
& A^{(i)}=\frac{3\left(\delta^{2} a^{(i)} b^{(i)}+8\right) k^{(M)}}{\left(\delta^{2} a^{(i)} b^{(i)}-8\right)\left(2 k^{(M)}+k^{(i)}\right)+c_{1}}, \\
& B^{(M)}=R^{3} \frac{\left(\delta^{2} a^{(i)} b^{(i)}-8\right)\left(k^{(i)}-k^{(M)}\right)-c_{2}}{\left(\delta^{2} a^{(i)} b^{(i)}-8\right)\left(2 k^{(M)}+k^{(i)}\right)+c_{1}}, \\
& c_{1}=8 \delta\left(a^{(i)} k^{(M)} k^{(i)}-b^{(i)}\right), \\
& c_{2}=4 \delta\left(a^{(i)} k^{(M)} k^{(i)}+2 b^{(i)}\right) .
\end{aligned}
$$

The expressions (47) and (48) together with (51)-(54) constitute the analytical exact solution to the benchmark problem under consideration. Remark that the temperature gradient inside the inclusion is uniform.

\subsection{Validation of the numerical procedure}

We now apply the numerical procedure presented in the last section to the benchmark problem described above. Precisely, a cube $\Omega$ of side length $l$ is cut out of the foregoing infinite matrix containing a spherical inclusion of radius $R$ with $l>2 R$. The center of the cube coincides with that of the spherical inclusion and, more specifically, the side length $l$ of the cube is set to be such that $R / l=1 / 4$ (see Fig. 5). On the surface $\partial \Omega$ of the cube, we prescribe the temperature field determined by the analytical exact expression (48) together with (52). In what follows, the remote temperature gradient $\mathbf{g}_{0}$ is chosen to be $\mathbf{g}_{0}=(0,0,100)^{T}$ with the component

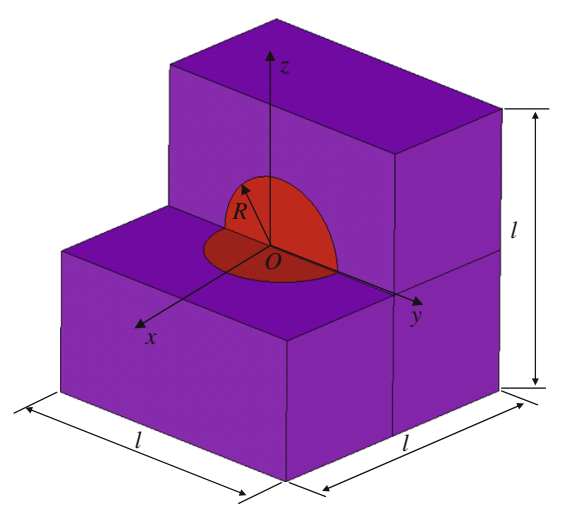

Fig. 5 Problem of a spherical inclusion inside a cubic matrix 
unit being $\mathrm{m}^{-1} \mathrm{~K}$, and the geometrical parameters $l$ and $\delta$ are set to be $l=0.04 \mathrm{~m}$ and $\delta=h / R=4 \times 10^{-4}$ except specified.

\subsubsection{Convergence analysis}

Simulations are performed on the cube described above and shown in Fig. 5. The whole domain of the cube is meshed with tetrahedra. The following two sets of bulk conductivities are used:

Set 1: $k^{(i)}=1 \mathrm{~W} \mathrm{~m}^{-1} \mathrm{~K}^{-1}, \quad \mathrm{k}^{(M)}=10 \mathrm{~W} \mathrm{~m}^{-1} \mathrm{~K}^{-1}$; Set $2: k^{(i)}=100 \mathrm{~W} \mathrm{~m}^{-1} \mathrm{~K}^{-1}, \quad k^{(M)}=10 \mathrm{~W} \mathrm{~m}^{-1} \mathrm{~K}^{-1}$.

In Set 1 , the conductivity $k^{(i)}$ of the inclusion is lower than the one $k^{(M)}$ of the matrix. In Set 2, the former is higher than the latter. The conductivity $k^{(0)}$ of the interphase involved in the expressions (7) of the interfacial parameters $a^{(i)}$ and $b^{(i)}$ varies numerically from $10^{-6}$ to $10^{6} \mathrm{~W} \mathrm{~m}^{-1} \mathrm{~K}^{-1}$, so that a wide class of imperfect interfaces ranging from the LC to $\mathrm{HC}$ interface are taken into account in our simulations.
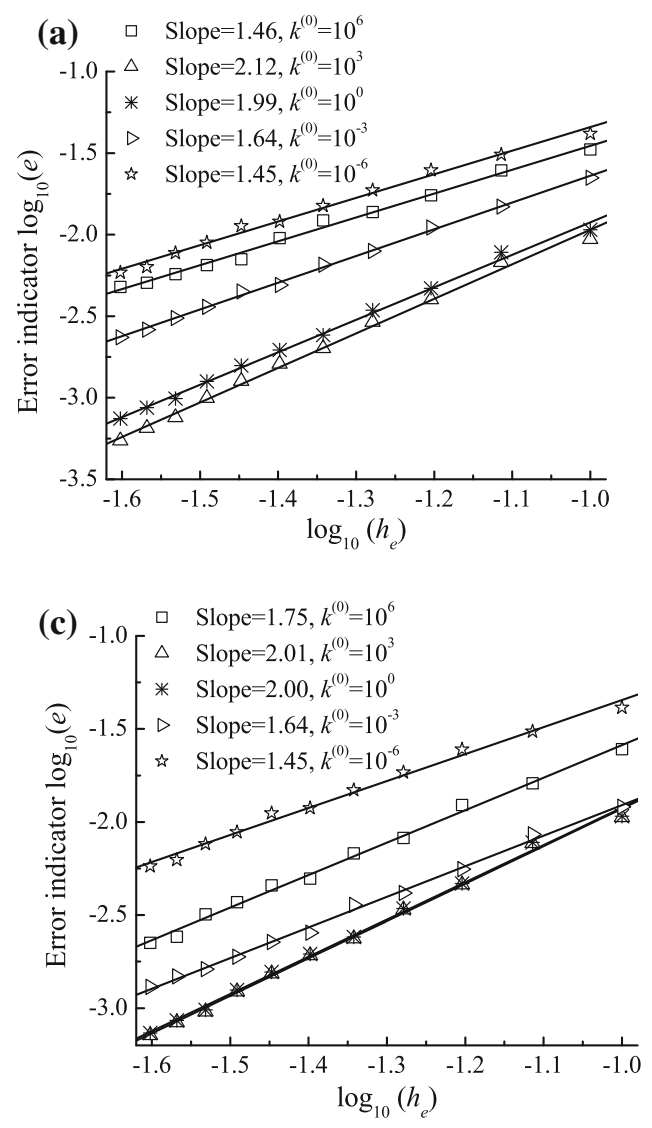

Fig. 6 Convergence analysis of the numerical results with respect to the exact analytical solution for two sets of bulk conductivities, two relative interphase thickness values and different interfacial conductivities.
The convergence analysis of the numerical procedure presented in the last section is carried out by considering different element sizes $h_{e}$. The global computational convergence is evaluated by using the following $L^{2}$-norm relative error indicator:

$e=\frac{\sqrt{\left.\frac{1}{V} \int_{\Omega}\left|\varphi^{h_{e}}-\varphi\right|^{2} d V+\frac{1}{S} \int_{\Gamma} \mid[[\varphi]]\right]^{h_{e}}-\left.[[\varphi]]\right|^{2} d S}}{\sqrt{\frac{1}{V} \int_{\Omega} \varphi^{2} d V+\frac{1}{S} \int_{\Gamma}[[\varphi]]^{2} d S}}$

where $\Gamma$ is the interface between the matrix and the spherical inclusion, $V$ stands for the volume of $\Omega, S$ represents the area of $\Gamma$, and $\varphi^{h_{e}}$ and $\varphi$ denote, respectively, the temperature fields calculated numerically and analytically.

The results of the convergence analysis are plotted in Fig. 6. It is seen that: (i) the relative error $e$ decreases when the mesh size $h_{e}$ diminishes; (ii) each numerical solution converges to the corresponding analytical exact solution when the mesh size decreases; (iii) the convergence rates are equal or close to the optimal value 2 when the interphase conductiv-
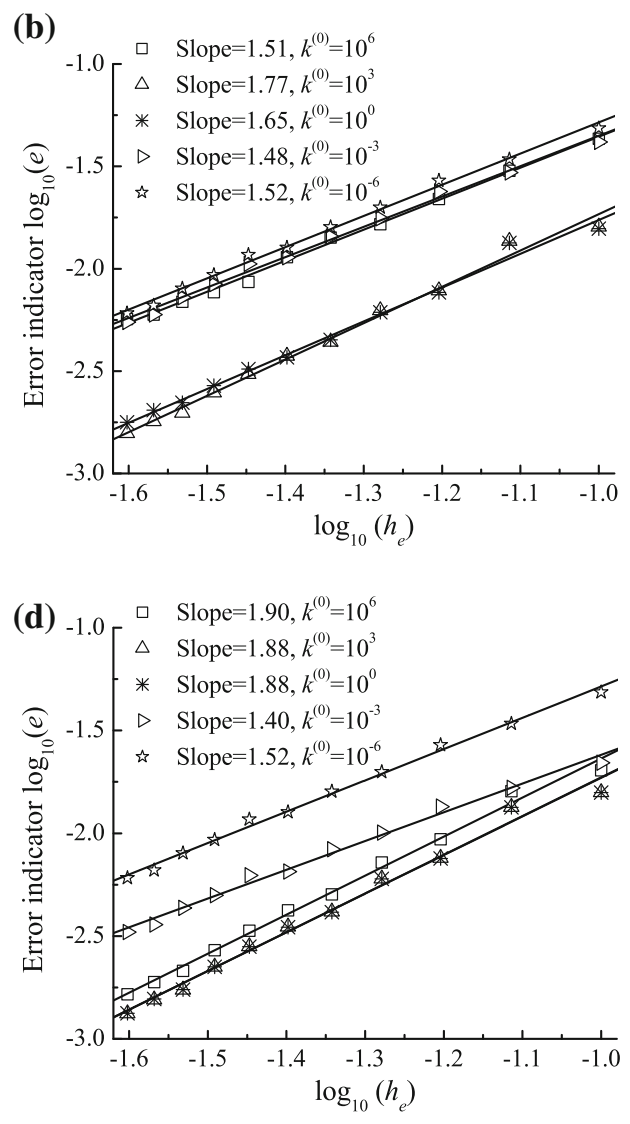

a Material set 1 with $\delta=4 \times 10^{-4}$. b Material set 2 with $\delta=4 \times 10^{-4}$. c Material set 1 with $\delta=4 \times 10^{-5}$. d Material set 2 with $\delta=4 \times 10^{-5}$ 
ity $k^{(0)}$ is not by several orders of magnitude different from the matrix and inclusion conductivities $k^{(M)}$ and $k^{(i)}$; (iv) Even when the interphase conductivity $k^{(0)}$ is several orders of magnitude higher or lower than the matrix and inclusion conductivities, the convergence rates are not smaller than 1.4. Thus, the convergence analysis shows that the proposed numerical procedure is efficient and robust.

In Fig. 6, two numerical values of the normalized interphase thickness $\delta=h / R$ are employed in the convergence analysis: $\delta=4 \times 10^{-4}$ and $\delta=4 \times 10^{-5}$. We observe that the convergence rates with a smaller value of $\delta$ are better than those with a larger value of $\delta$. The interphase thickness $h$ as a parameter involved in the weak form (28) and the expression (43) of the interface conductivity matrix $\mathbf{K}_{s}$ plays an important role from the numerical standpoint when the contrast between the interphase conductivity $k^{(0)}$ and the matrix and inclusion conductivities $k^{(M)}$ and $k^{(i)}$ is very high. Indeed, when $k^{(0)}>>k^{(M)}$ and $k^{(0)}>>k^{(i)}$, it follows from (7) that

$h b^{(i)} / 2 \approx h k^{(0)}, \quad 2 /\left(h a^{(i)}\right) \approx \frac{2 k^{(i)}}{h\left(k^{(i)}+k^{(M)}\right)}$.

Thus, it is seen from (41) and (43) that, when the value of $h$ is sufficiently small, $h$ acts as a penalty parameter such that the solution of (41) gives $[[\varphi]]_{i} \approx 0$ as indicated by (10), which means that the temperature jump across the interface $\Gamma_{i}$ is zero to within a given error.

If $k^{(0)}<<k^{(M)}$ and $k^{(0)}<<k^{(i)}$, we have

$h b^{(i)} / 2 \approx 0, \quad 2 /\left(h a^{(i)}\right) \approx-\frac{k^{(0)}}{h}$

when $h$ is sufficiently small, and the relations (8) and (9) hold. In this case, the second term in the left-hand member of the weak form (28) and the term involving $b^{(i)}$ in the expression (43) of $\mathbf{K}_{s}$ are numerically negligible.

In the LC and HC cases, as indicated by (8)-(9) and (10)(11), error of the interfacial jump relations is of the same order as $h$. Thus, when the interphase thickness $h$ increases, error of the interfacial jumps becomes larger and the convergence rates in Fig. 6 are smaller.

For the following discussions, it is convenient to introduce the interfacial parameters:

$\alpha^{(i)}=h a^{(i)} / 2=h\left(\frac{1}{2 k^{(M)}}+\frac{1}{2 k^{(i)}}-\frac{1}{k^{(0)}}\right)$,

$\beta^{(i)}=h b^{(i)} / 2=h\left(k^{(0)}-k^{(M)} / 2-k^{(i)} / 2\right)$.

\subsubsection{Lowly conducting interfaces}

Now, we examine if the numerical procedure proposed can correctly and efficiently capture the main physical aspects of thermal conduction when $k^{(0)}<<k^{(M)}$ and $k^{(0)}<<k^{(i)}$. In this case, our general imperfect interface model reduces to Kapitza's thermal resistance model to within an error of order $0(h)$. The whole domain illustrated in Fig. 5 is meshed with 300,000 tetrahedra and 64,000 nodes. For simulations, only one set of bulk material parameters, namely Set 1 given above, is used, the normalized thickness $\delta$ of the interphase is taken to be $\delta=4 \times 10^{-4}$, and the interphase conductivity $k^{(0)}$ ranging from $10^{-6}$ to $10^{-2} \mathrm{~W} \mathrm{~m}^{-1} \mathrm{~K}^{-1}$ is considered. The corresponding values of the interfacial parameters $\alpha^{(i)}$ and $\beta^{(i)}$ defined by (56) are listed in Table 1 . Note that the numerical value of $\beta^{(i)}$ is comparatively very small.

The analytic and numerical results of the temperature and normal heat flux along the $z$-axis for different values of $k^{(0)}$ are plotted and compared in Fig. 7. A good agreement is observed between them. Concerning the numerical results, as expected, the temperature is discontinuous across the interface, and this discontinuity increases when $k^{(0)}$ decreases. At the same time, the normal heat flux presents so small numerical jump that it can be considered as being continuous across the interface. Figure 8 shows the influence of $k^{(0)}$ on the temperature fields in the plane $x=0$ by nephograms. The effect of Kapitza's thermal resistance on the continuity of the temperature field is clearly captured and quite similar to that reported by Yvonnet et al. [5]. The oscillations of the contours in the case with $k^{(0)}=10^{-6} \mathrm{~W} \mathrm{~m}^{-1} \mathrm{~K}^{-1}$ are mainly caused by the post-processing process which does not account for the discontinuity in the cut elements. In conclusion, by appropriately choosing the numerical values of the parameters in the general imperfect interface model, the proposed numerical procedure can efficiently deal with the LC interface and reflect Kapitza's thermal resistance effect.

\subsubsection{Highly conducting interfaces}

To examine the capacity of the proposed numerical procedure to deal with the effects of a HC interface, the same finite element model and the same geometrical and material parameters as in the case of LC interfaces are adopted here,

Table 1 Numerical values of the parameters used in simulating lowly conducting interfaces

\begin{tabular}{llll}
\hline$k^{(0)}\left(\mathrm{W} \mathrm{m}^{-1} \mathrm{~K}^{-1}\right)$ & $\delta$ & $\alpha^{(i)}$ & $\beta^{(i)}$ \\
\hline $10^{-6}$ & $4 \times 10^{-4}$ & -1.00 & $-5.50 \times 10^{-6}$ \\
$10^{-5}$ & $4 \times 10^{-4}$ & -0.10 & $-5.50 \times 10^{-6}$ \\
$10^{-4}$ & $4 \times 10^{-4}$ & -0.01 & $-5.50 \times 10^{-6}$ \\
$10^{-3}$ & $4 \times 10^{-4}$ & $-9.99 \times 10^{-4}$ & $-5.50 \times 10^{-6}$ \\
$10^{-2}$ & $4 \times 10^{-4}$ & $-9.95 \times 10^{-5}$ & $-5.49 \times 10^{-6}$ \\
\hline
\end{tabular}



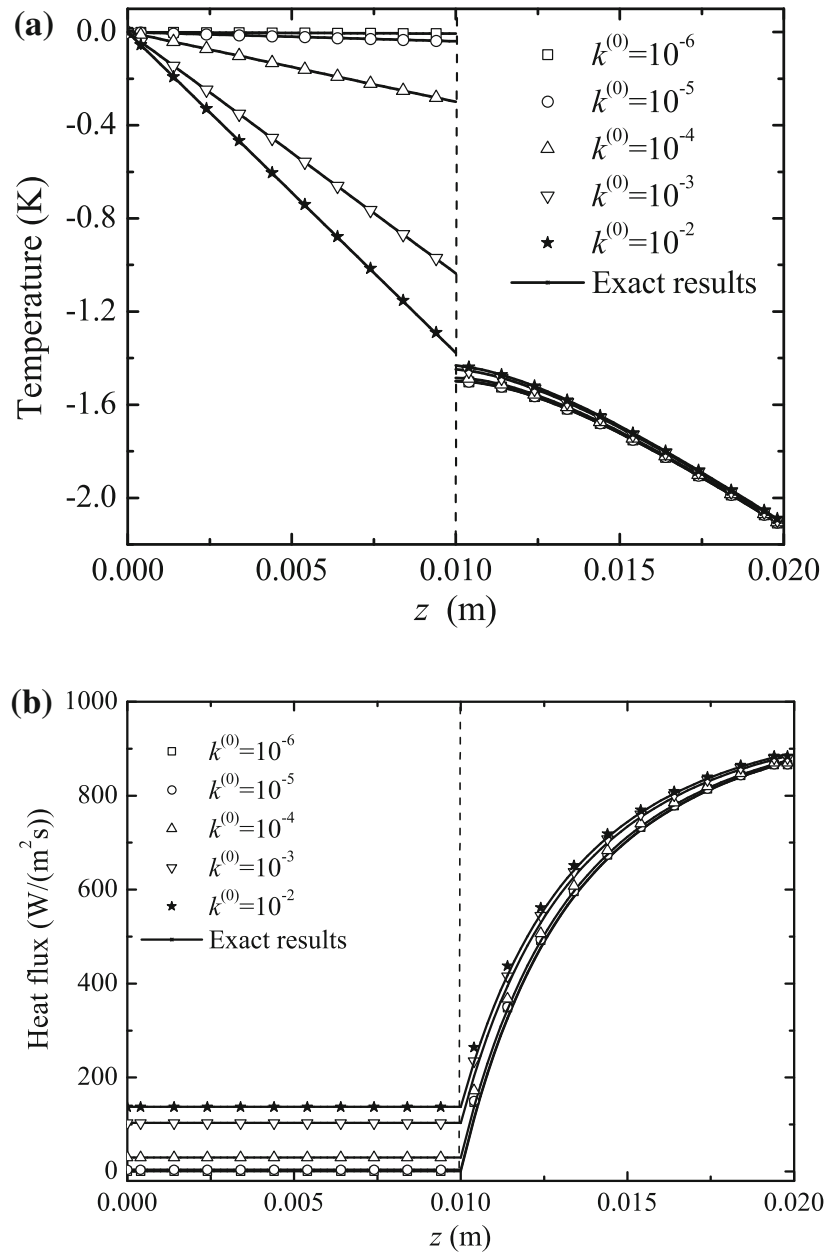

Fig. 7 Comparison of the theoretical and numerical results for the temperature and normal heat flux along the $z$-axis with $x=0$ and $y=0$ in the benchmark problem of a spherical inclusion inside a cube with different small values of $k^{(0)}$. a Temperature. b Heat flux
Table 2 Numerical values of the parameters used in simulating highly conducting interfaces

\begin{tabular}{llll}
\hline$k^{(0)}\left(\mathrm{W} \mathrm{m}^{-1} \mathrm{~K}^{-1}\right)$ & $\delta$ & $\alpha^{(i)}$ & $\beta^{(i)}$ \\
\hline $10^{2}$ & $4 \times 10^{-4}$ & $5.40 \times 10^{-7}$ & $9.45 \times 10^{-5}$ \\
$10^{3}$ & $4 \times 10^{-4}$ & $5.49 \times 10^{-7}$ & $9.95 \times 10^{-4}$ \\
$10^{4}$ & $4 \times 10^{-4}$ & $5.50 \times 10^{-7}$ & 0.01 \\
$10^{5}$ & $4 \times 10^{-4}$ & $5.50 \times 10^{-7}$ & 0.10 \\
$10^{6}$ & $4 \times 10^{-4}$ & $5.50 \times 10^{-7}$ & 1.00 \\
\hline
\end{tabular}

except the interphase conductivity $k^{(0)}$ ranging this time from $10^{2}$ to $10^{6} \mathrm{~W} \mathrm{~m}^{-1} \mathrm{~K}^{-1}$. The corresponding numerical values of the interfacial parameters $\alpha^{(i)}$ and $\beta^{(i)}$ are listed in Table 2. It is seen that the numerical value of $\alpha^{(i)}$ is comparatively very small.

For the values of $k^{(0)}$ listed in Table 2, the numerical results obtained for the temperature and normal heat flux along the $z$-axis are plotted and compared with the corresponding analytical exact results in Fig. 9. This comparison shows an excellent agreement between the numerical and analytical results. In particular, the continuity of the temperature and the jump of the normal heat flux across the interface are correctly captured by the numerical results. As expected, the jump of the normal heat flux becomes accentuated when the value of $k^{(0)}$ (or $\beta^{(i)}$ ) augments. The temperature fields with $k^{(0)}=10^{2}$ and $10^{6} \mathrm{~W} \mathrm{~m}^{-1} \mathrm{~K}^{-1}$ in the plane $x=0$ are given as nephograms in Figure 10. Comparison of those two fields shows that $k^{(0)}$ can significantly affect the temperature field. In conclusion, by properly choosing the values of the interfacial parameters, the proposed numerical procedure is able to correctly and efficiently treat $\mathrm{HC}$ interfaces. (a)

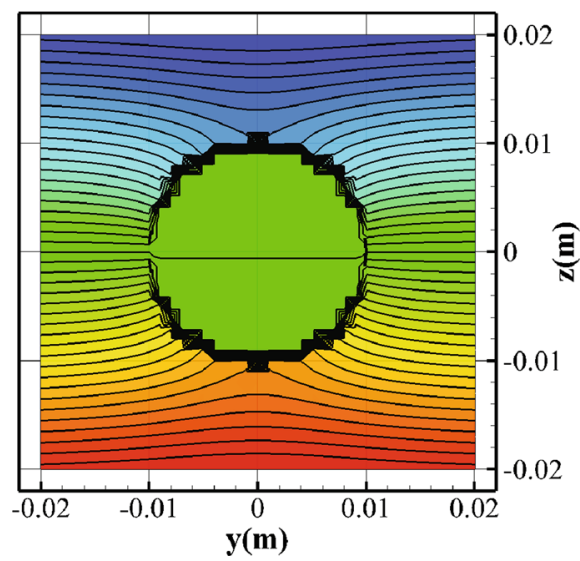

(b)

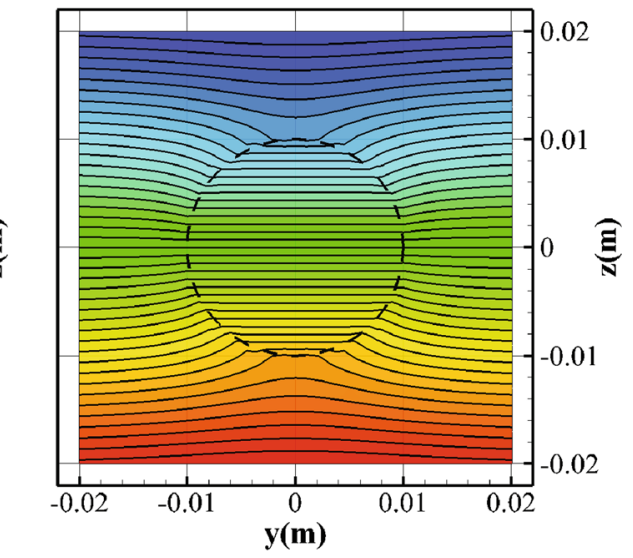

Fig. 8 Temperature fields in the plane $x=0$ with $k^{(0)}=10^{-6}$ and $10^{-2} \mathrm{~W} \mathrm{~m}^{-1} \mathrm{~K}^{-1}$. a $k^{(0)}=10^{-6} \mathrm{~W} \mathrm{~m}^{-1} \mathrm{~K}^{-1} \cdot \mathbf{b} k^{(0)}=10^{-2} \mathrm{~W} \mathrm{~m}^{-1} \mathrm{~K}^{-1}$ 

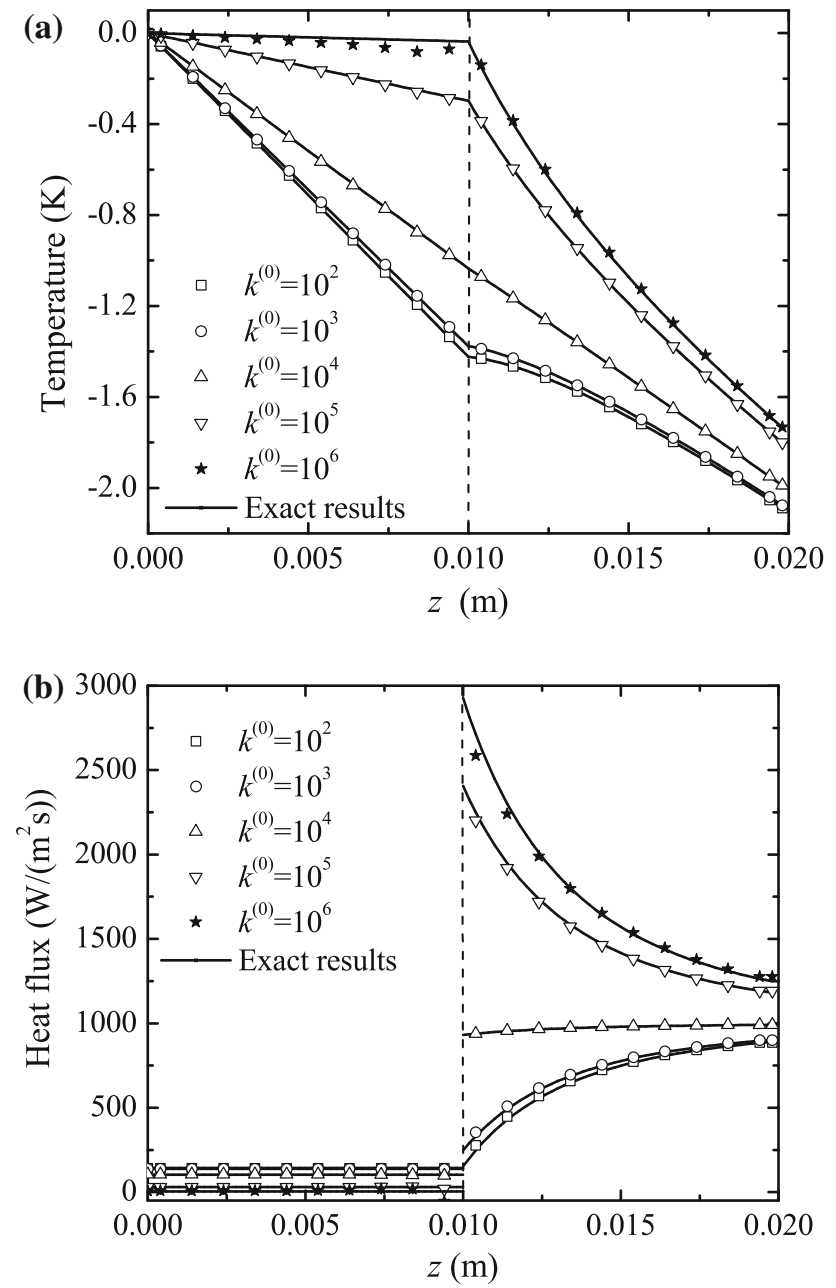

Fig. 9 Comparison of the theoretical and numerical results for the temperature and normal heat flux along the $z$-axis with $x=0$ and $y=0$ in the benchmark problem of a spherical inclusion inside a cube with different large values of $k^{(0)}$. a Temperature. b Heat flux
Table 3 Numerical values of the parameters used in simulating intermediate interfaces

\begin{tabular}{llll}
\hline$k^{(0)}\left(\mathrm{W} \mathrm{m}^{-1} \mathrm{~K}^{-1}\right)$ & $\delta$ & $\alpha^{(i)}$ & $\beta^{(i)}$ \\
\hline 0.15 & $4 \times 10^{-4}$ & $-6.12 \times 10^{-6}$ & $-5.35 \times 10^{-6}$ \\
1.5 & $4 \times 10^{-4}$ & $-1.17 \times 10^{-7}$ & $-4.00 \times 10^{-6}$ \\
15 & $4 \times 10^{-4}$ & $4.83 \times 10^{-7}$ & $9.50 \times 10^{-6}$ \\
0.15 & 0.2 & $-1.22 \times 10^{-2}$ & $-1.07 \times 10^{-2}$ \\
1.5 & 0.2 & $-2.33 \times 10^{-4}$ & $-8.00 \times 10^{-3}$ \\
15 & 0.2 & $9.67 \times 10^{-4}$ & $1.90 \times 10^{-2}$ \\
\hline
\end{tabular}

\subsubsection{Intermediate cases}

When the value of the interphase conductivity $k^{(0)}$ is neither very high nor very low or when its thickness $h$ is not sufficiently small, the interface characterized by the relations (3) and (4) are qualified as intermediate. In such cases, both the temperature and the normal heat flux across the interface are in general discontinuities. To test the ability of the proposed numerical procedure to treat this situation, the same finite element model as above is adopted and the values of the matrix and inclusion conductivities are taken to be given by Set 1 . In addition, the value of $k^{(0)}$ varies from $10^{-1}$ to 10 $\mathrm{W} \mathrm{m}{ }^{-1} \mathrm{~K}^{-1}$ and two values of the normalized phase thickness $\delta$, namely $\delta=4 \times 10^{-4}$ and $\delta=0.2$, are considered. In Table 3, the corresponding numerical values of $\alpha^{(i)}$ and $\beta^{(i)}$ are provided.

In Fig. 11, the numerical and analytical results for the temperature and normal heat flux along the $z$-axis are plotted for $\delta=4 \times 10^{-4}$ and for three values of $k^{(0)}$. Since the corresponding values of $\alpha^{(i)}$ and $\beta^{(i)}$ are very small, the presence of the imperfect interface has negligible influence on the temperature field so that all the numerical and analytical results are almost identical to each other. (a)

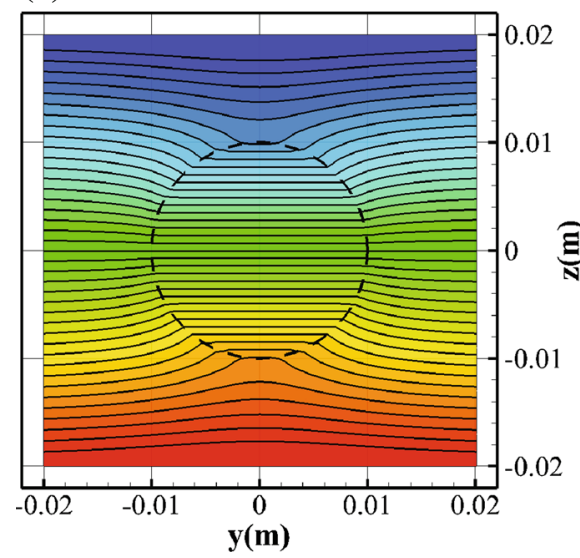

(b)

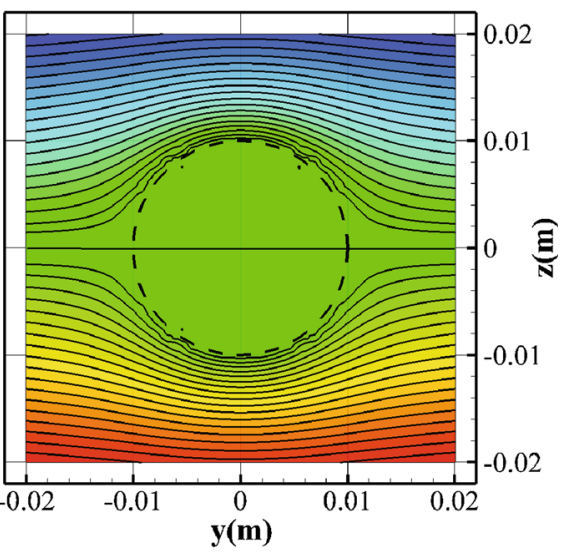

Fig. 10 Temperature fields in the plane $x=0$ with $k^{(0)}=10^{2}$ and $10^{6} \mathrm{~W} \mathrm{~m}^{-1} \mathrm{~K}^{-1}$. a $k^{(0)}=10^{2} \mathrm{~W} \mathrm{~m}^{-1} \mathrm{~K}^{-1} \cdot \mathbf{b} k^{(0)}=10^{6} \mathrm{~W} \mathrm{~m}^{-1} \mathrm{~K}^{-1}$ 

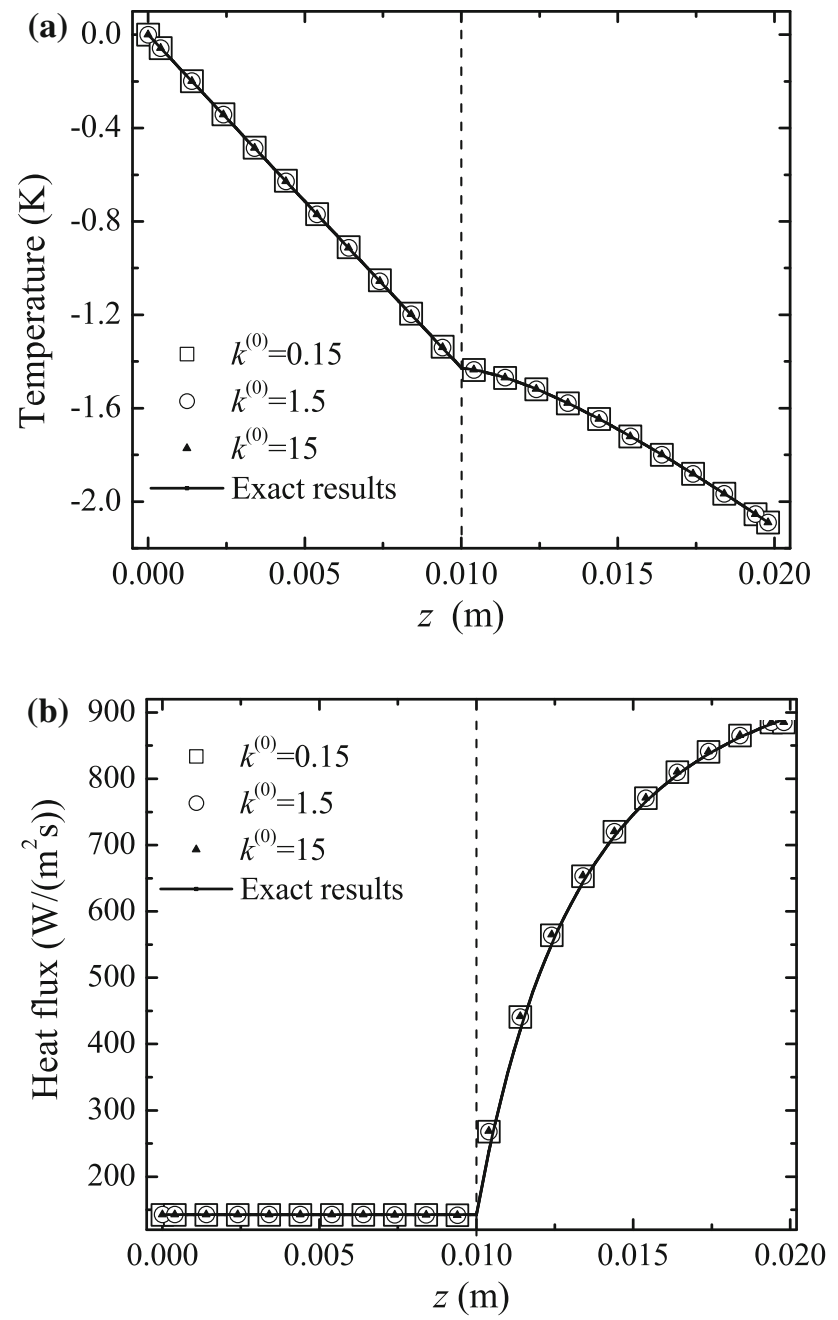

Fig. 11 Comparison of the theoretical and numerical results for the temperature and normal heat flux along the $z$-axis with $x=0$ and $y=0$ in the benchmark problem of a spherical inclusion inside a cube with the value of $k^{(0)}$ being not far from those of $k^{(i)}$ and $k^{(M)}$ and with $h$ being relatively very small. a Temperature. b Heat flux

In Fig. 12, we plot the numerical and analytical results for the temperature and normal heat flux along the $z$-axis for $\delta=0.2$. A good concordance is observed between them. The numerical results are successful in capturing the temperature and normal heat flux jumps across the interface. These discontinuities become more pronounced when the values of $\alpha^{(i)}$ and $\beta^{(i)}$ increase.

The foregoing discussions clearly indicate that the proposed numerical method is capable of treating various intermediate cases where both the weak and strong discontinuities occur.

\subsubsection{Effects of the interphase thickness}

As has been noted above, the interphase thickness $h$ affects theoretical and numerical results in an important way. To elucidate its effects, the foregoing finite element model
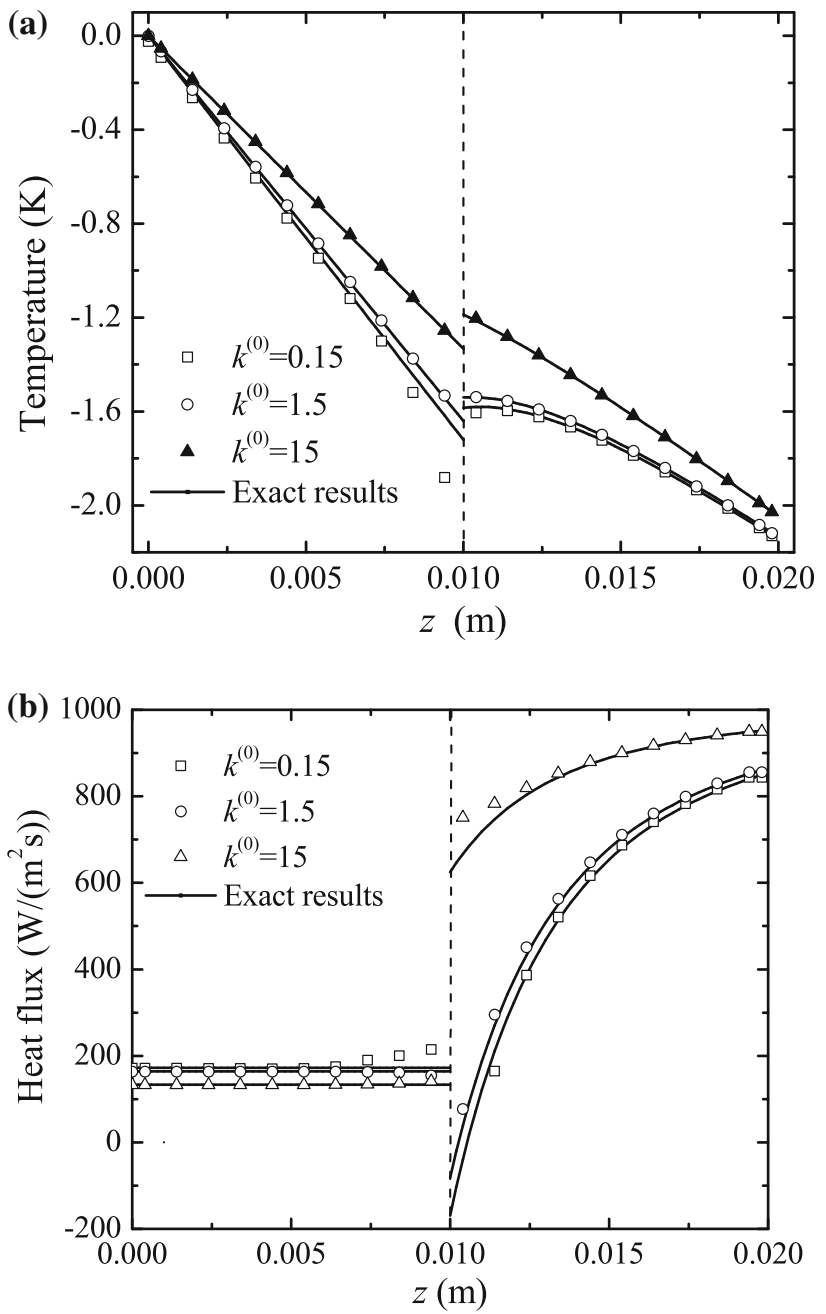

Fig. 12 Comparison of the theoretical and numerical results for the temperature and normal heat flux along the $z$-axis with $x=0$ and $y=0$ in the benchmark problem of a spherical inclusion inside a cube with the value of $k^{(0)}$ being not far from those of $k^{(i)}$ and $k^{(M)}$ but with $h$ being no longer very small. aTemperature. b Heat flux

is employed. The bulk conductivities are fixed as $k^{(i)}=$ $1 \mathrm{~W} \mathrm{~m}^{-1} \mathrm{~K}^{-1}$ and $k^{(M)}=10 \mathrm{~W} \mathrm{~m}^{-1} \mathrm{~K}^{-1}$ while the interphase conductivity $k^{(0)}$ ranges from $10^{-6}$ to $10^{6} \mathrm{~W} \mathrm{~m}^{-1} \mathrm{~K}^{-1}$ and the normalized interphase thickness $\delta=h / R$ varies from $10^{-5}$ to $10^{-3}$. The corresponding values of $\alpha^{(i)}$ and $\beta^{(i)}$ are listed in Table 4. The numerical results and their comparison with the associated theoretical results are provided in Fig. 13. As expected, in all the intermediate cases, the jumps in temperature and normal heat flux increase when $\delta$ increases. When the Kapitza or HC interface model applies, the temperature or normal heat flux discontinuity become more remarkable when $\delta$ is larger.

\subsection{Thermal conduction in a particulate composite}

The numerical procedure elaborated in the last section has been validated above with the help of a benchmark problem. 
Table 4 Numerical values of the parameters used in simulating effects of the interphase thickness $\delta$

\begin{tabular}{llll}
\hline & $k^{(0)}\left(\mathrm{W} \mathrm{m}^{-1} \mathrm{~K}^{-1}\right)$ & $\alpha^{(i)}$ & $\beta^{(i)}$ \\
\hline$\delta=10^{-5}$ & $10^{-6}$ & $-2.50 \times 10^{-2}$ & $-1.38 \times 10^{-7}$ \\
& $10^{-3}$ & $-2.50 \times 10^{-5}$ & $-1.37 \times 10^{-7}$ \\
& $10^{0}$ & $-1.13 \times 10^{-8}$ & $-1.13 \times 10^{-7}$ \\
& $10^{3}$ & $1.37 \times 10^{-8}$ & $2.49 \times 10^{-5}$ \\
& $10^{6}$ & $1.38 \times 10^{-8}$ & 0.0250 \\
$\delta=10^{-4}$ & $10^{-6}$ & -0.25 & $-1.38 \times 10^{-6}$ \\
& $10^{-3}$ & $-2.50 \times 10^{-4}$ & $-1.37 \times 10^{-6}$ \\
& $10^{0}$ & $-1.13 \times 10^{-7}$ & $-1.13 \times 10^{-6}$ \\
& $10^{3}$ & $1.37 \times 10^{-7}$ & $2.49 \times 10^{-4}$ \\
& $10^{6}$ & $1.38 \times 10^{-7}$ & 0.25 \\
& $10^{-6}$ & -2.50 & $-1.38 \times 10^{-5}$ \\
& $10^{-3}$ & $-2.50 \times 10^{-3}$ & $-1.37 \times 10^{-5}$ \\
& $10^{0}$ & $-1.13 \times 10^{-6}$ & $-1.13 \times 10^{-5}$ \\
& $10^{3}$ & $1.37 \times 10^{-6}$ & $2.50 \times 10^{-3}$ \\
& $10^{6}$ & $1.38 \times 10^{-6}$ & 2.50 \\
\hline
\end{tabular}

Now, we apply it to solve the problem of steady thermal conduction in a composite consisting of a matrix in which multiple spherical and non-spherical inclusions are embedded. As an example and for simplicity, consider a representative volume element (RVE) $\Omega$ of the composite, which corresponds to a cubic matrix with eight inclusions (Fig. 14). Relative to the system of Cartesian coordinates $(x, y, z)$, each inclusion is characterized by

$\left|x-x_{c}^{(j)}\right|^{p}+\left|y-y_{c}^{(j)}\right|^{p}+\left|z-z_{c}^{(j)}\right|^{p}=a_{j}^{p}$

where $\left(x_{c}^{(j)}, y_{c}^{(j)}, z_{c}^{(j)}\right)$ are the coordinates of the center of the $j$ th inclusion, $p \geqslant 1$ and $a_{j}$ is a positive constant which is required to be such that any two inclusions do not contact each other and each inclusion is completely included inside the matrix.

Two configurations are numerically investigated. The locations and geometric parameters of the inclusions for every configuration are specified in Table 5. Figure 14 illustrates configuration 1 . The interface between the matrix and each inclusion is imperfect and described by the jump relations (3) and (4). The interfacial and bulk parameters used are: $k^{(0)}=10^{-6}, 5$ or $10^{6} \mathrm{~W} \mathrm{~m}^{-1} \mathrm{~K}^{-1}, k^{(i)}=$ $1 \mathrm{~W} \mathrm{~m}^{-1} \mathrm{~K}^{-1}, k^{(M)}=10 \mathrm{~W} \mathrm{~m}^{-1} \mathrm{~K}^{-1}$ and $h=10^{-5} l$ with $l=0.04 \mathrm{~m}$ being the side length of the cubic matrix. On the surface $\partial \Omega$ of the RVE $\Omega$, we prescribe the uniform temperature gradient boundary condition $\varphi=\bar{\varphi}=-\mathbf{g}_{0} \cdot \mathbf{x}$ for $\mathbf{x} \in$ $\partial \Omega$ and with $\mathbf{g}_{0}=(0,0,100)^{T}$ whose component unit is $\mathrm{Km}^{-1}$.
Figures 15, 16 and 17 show the temperature distribution in three planes normal to the axis $y$ for configuration 1 with $k^{(0)}=10^{-6}, 5$ and $10^{6} \mathrm{~W} \mathrm{~m}^{-1} \mathrm{~K}^{-1}$. The effects of lowly and $\mathrm{HC}$ imperfect interfaces on the temperature distribution are clearly observed. From 15, 16 and 17, we see that the temperature distribution inside all inclusion is much less uniform in the case of $k^{(0)}=5 \mathrm{~W} \mathrm{~m}^{-1} \mathrm{~K}^{-1}$ than the cases of $k^{(0)}=10^{-6}$ and $10^{6} \mathrm{~W} \mathrm{~m}^{-1} \mathrm{~K}^{-1}$. Further, the temperature distribution over each inclusion is more uniform in the case of $k^{(0)}=10^{-6} \mathrm{~W} \mathrm{~m}^{-1} \mathrm{~K}^{-1}$ than in the case of $k^{(0)}=10^{6} \mathrm{~W} \mathrm{~m}^{-1} \mathrm{~K}^{-1}$. Even though the matrix is finite and multiple inclusions are present, the temperature distribution over each inclusion is almost uniform in the case of $k^{(0)}=10^{-6} \mathrm{~W} \mathrm{~m}^{-1} \mathrm{~K}^{-1}$. However, the numerical values of the temperature field indicate that the gradient of the temperature field in each inclusion is not exactly uniform.

Figures 18, 19 and 20 illustrate the temperature distribution in three planes normal to the axis $y$ for configuration 2 where non-spherical inclusions are involved and $k^{(0)}=10^{-6}, 5$ and $10^{6} \mathrm{~W} \mathrm{~m}^{-1} \mathrm{~K}^{-1}$. Once more, we see that the lowly and $\mathrm{HC}$ imperfect interfaces considerably affect the temperature distribution. In particular, the lowly or HC interface tends to render the temperature inside an inclusion more uniform. In addition, the temperature field is influenced by the geometrical form of an inclusion. As expected, the temperature field inside each inclusion is non uniform. However, this non uniformity is quite weak in the cases of lowly and $\mathrm{HC}$ interfaces.

\section{Closing remarks}

In this work, a versatile interface model for thermal conduction has been considered and numerically implemented with the help of the XFEM and the LSM. The interface model in question is rigorously derived from a physically meaningful configuration and includes as special cases the classical Kapitza's resistance and HC imperfect interface models. In addition, this model is quite simple in the sense that it involves only two scalar parameters bearing clear physical interpretations. The weak form and numerical procedure elaborated for the interface model in question can be viewed as extending those presented by Yvonnet et al. [5,12] for the Kapitza and HC interface models. Validated with the aid of a benchmark problem, the numerical procedure provided in the this work is an efficient tool for the modelling and simulating the effects of a wide class of thermal imperfect interfaces in heterogeneous materials and structures.

Transport phenomena are numerous and play an important role in mechanics and physics. From a mathematical point of view, they are similar to each other, so that thermal conduction can be considered as a prototype of transport 
Fig. 13 Effects of the normalized interphase thickness $\delta$ on the temperature and normal heat flux along the $z$-axis with $x=0$ and $y=0$ in the benchmark problem of a spherical inclusion inside a cube with different values of $k^{(0)}$. a Temperature with $\delta=10^{-5}$. b Heat flux with $\delta=10^{-5}$. c Temperature with $\delta=10^{-4}$. d Heat flux with $\delta=10^{-4}$. e Temperature with $\delta=10^{-3}$. f Heat flux with $\delta=10^{-3}$
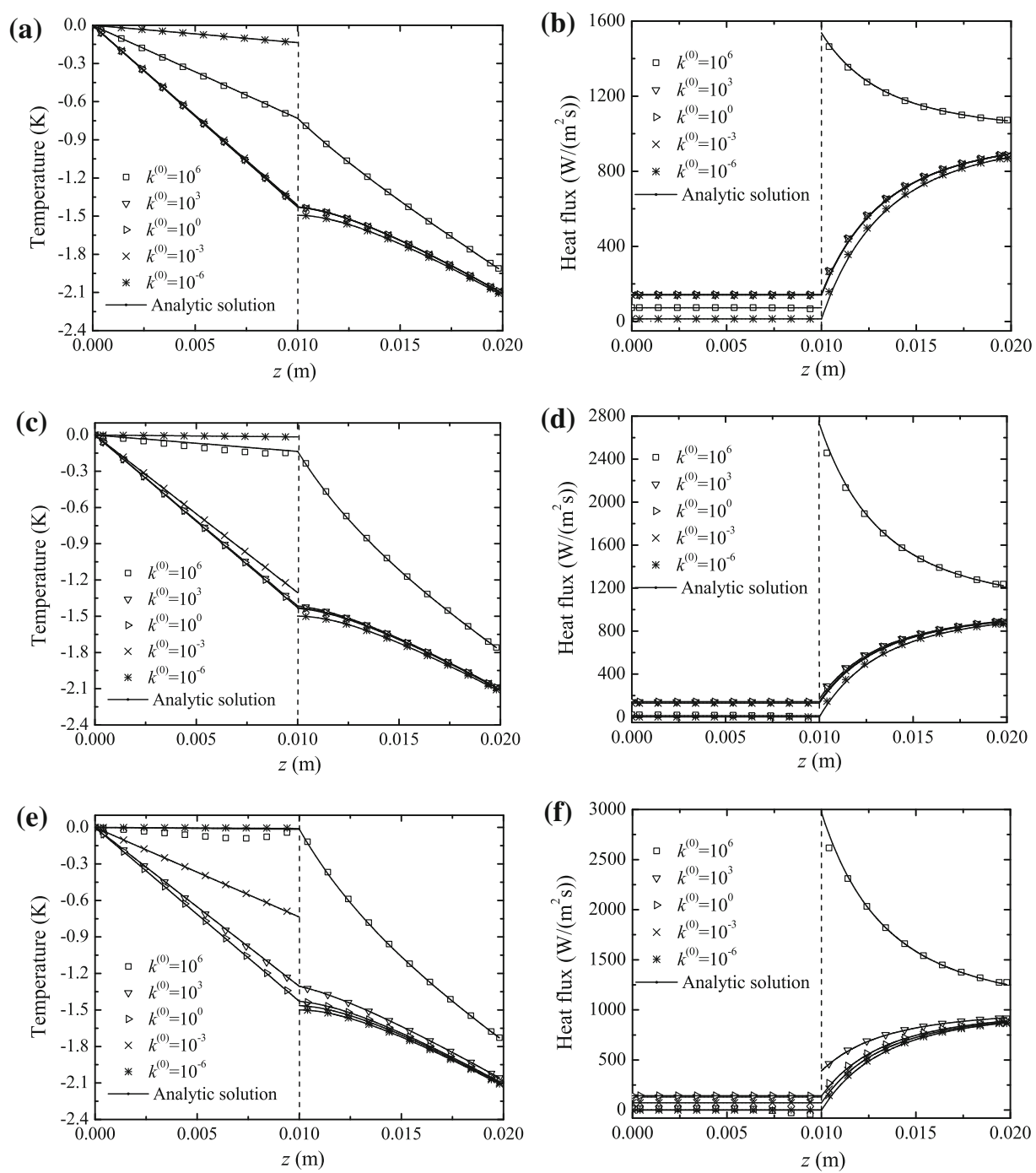

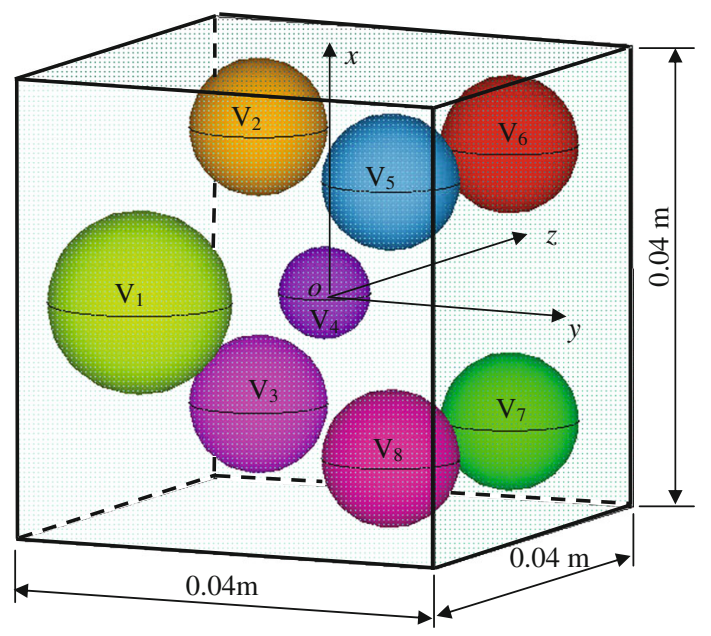

Fig. 14 Representative volume element (RVE) $\Omega$ of the particulate composite with 8 spherical inclusions

phenomena. Thus, when care is taken at the level of physics, the interface model considered and the numerical procedure
Table 5 Positions and sizes of the inclusions inside the RVE of the composite (unit: $\mathrm{mm}$ )

\begin{tabular}{lllll}
\hline Inclusions & $\begin{array}{l}\text { Config } 1 \text { and } 2 \\
\left(x_{c}, y_{c}, z_{c}\right)\end{array}$ & $a$ & Config 1 & Config 2 \\
\cline { 3 - 5 } & $(0,-11,-11)$ & 8 & 2 & $p$ \\
\hline V1 & $(12,-12,12)$ & 6 & 2 & 2 \\
V2 & $(-12,-12,12)$ & 6 & 2 & 5 \\
V3 & $(0,0,0)$ & 4 & 2 & 5 \\
V4 & $(12,12,-12)$ & 6 & 2 & 2 \\
V5 & $(12,12,12)$ & 6 & 2 & 4 \\
V6 & $(-12,12,12)$ & 6 & 2 & 4 \\
V7 & $(-12,12,-12)$ & 6 & 2 & 3 \\
V8 & & & & 3 \\
\hline
\end{tabular}

elaborated in the present work are expected to be transposable to other transport phenomena such as electrical conduction, diffusion, dielectricity and magnetism. In addition, due to the fact that anti-plane elasticity is mathematically identical to 
(a)

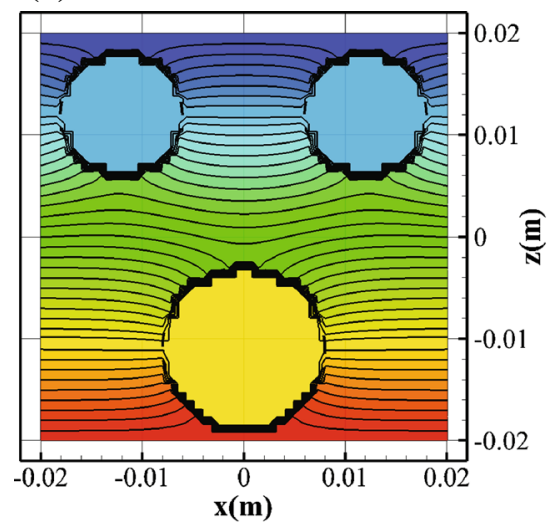

(b)

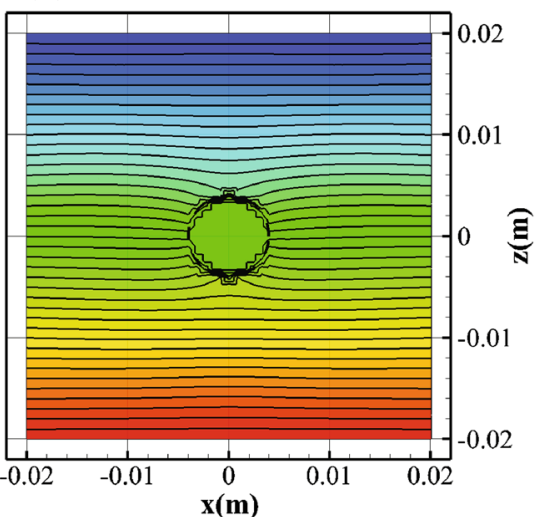

(c)

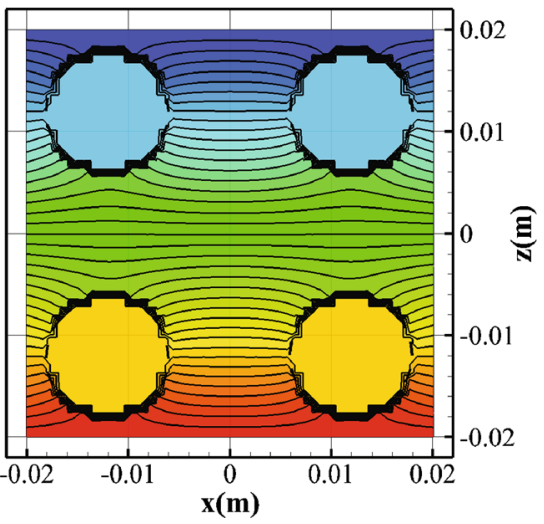

Fig. 15 Distribution of the temperature field in the RVE for configuration 1 and with $k^{(0)}=10^{-6} \mathrm{~W} \mathrm{~m}^{-1} \mathrm{~K}^{-1}$. a $y=-0.012 \mathrm{~m}$. b $y=0 \mathrm{~m}$. c $y=0.012 \mathrm{~m}$

(a)

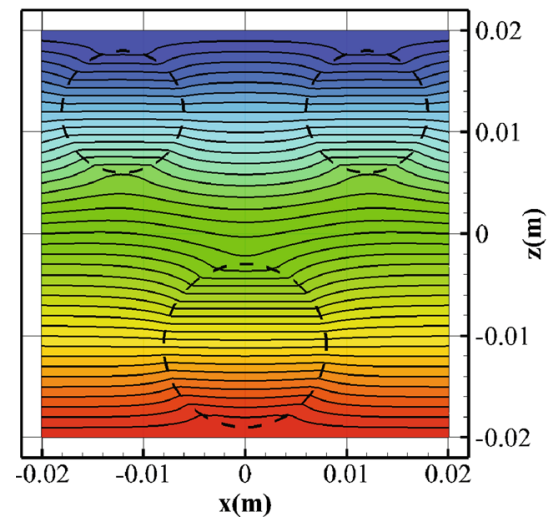

(b)

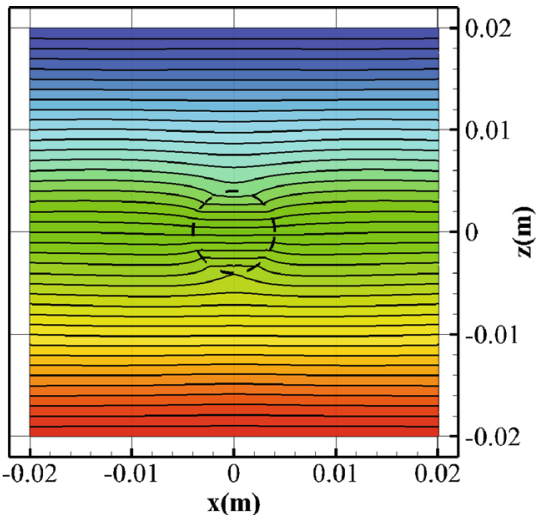

(c)

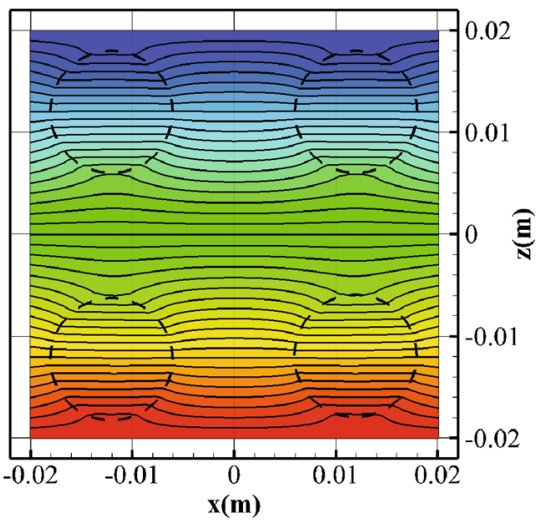

Fig. 16 Distribution of the temperature field in the RVE for configuration 1 and with $k^{(0)}=5 \mathrm{~W} \mathrm{~m}^{-1} \mathrm{~K}^{-1}$. a $y=-0.012 \mathrm{~m}$. b $y=0 \mathrm{~m}$. c $y=0.012 \mathrm{~m}$

(a)

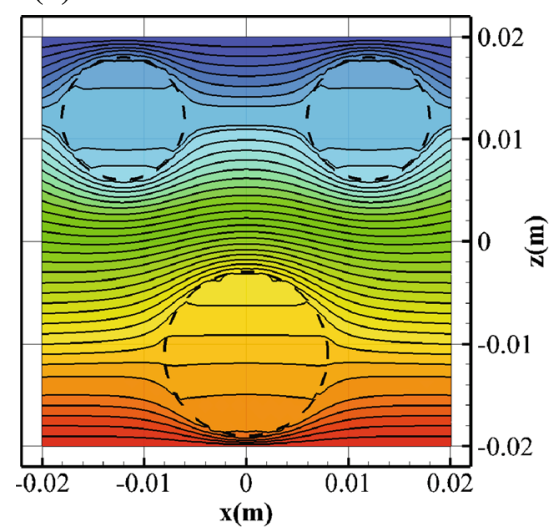

(b)

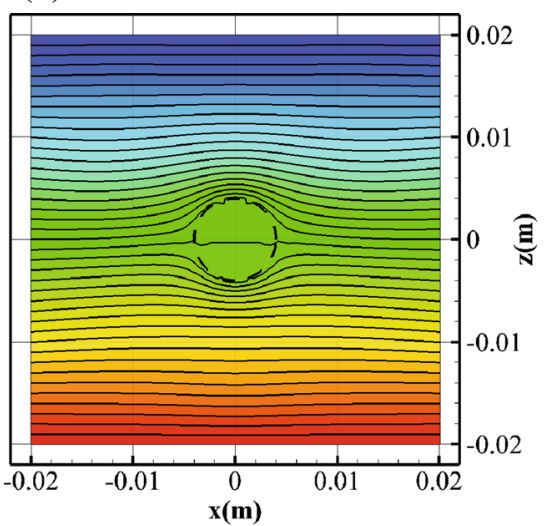

(c)

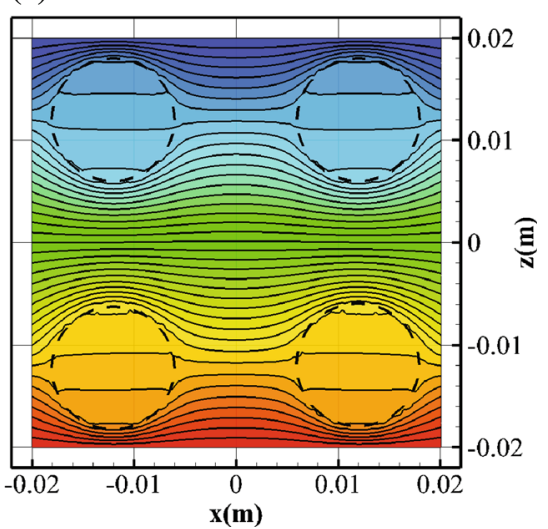

Fig. 17 Distribution of the temperature field in the RVE for configuration 1 and with $k^{(0)}=10^{6} \mathrm{~W} \mathrm{~m}^{-1} \mathrm{~K}^{-1}$. a $y=-0.012 \mathrm{~m}$. b $y=0 \mathrm{~m}$. c $y=0.012 \mathrm{~m}$ 
(a)

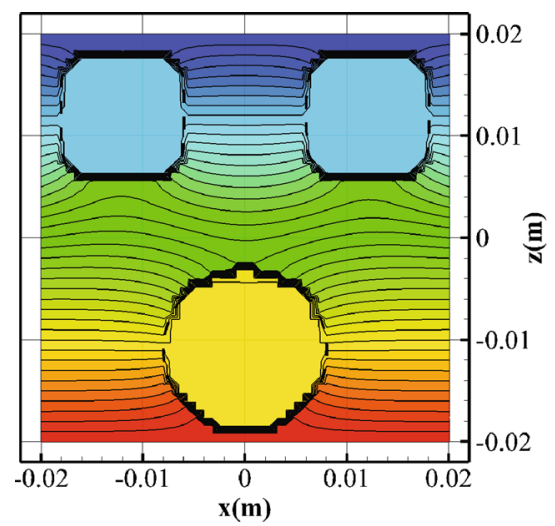

(b)

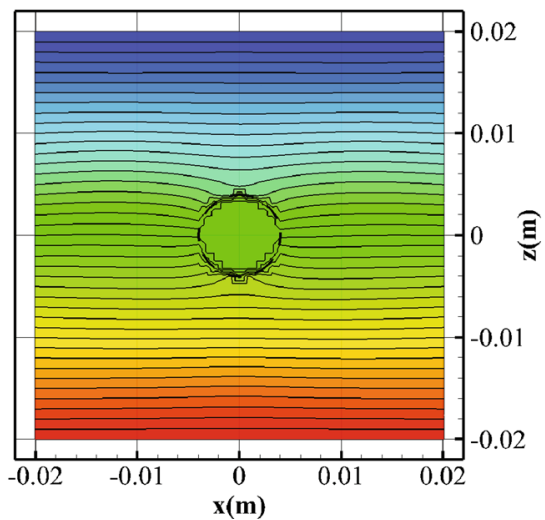

(c)

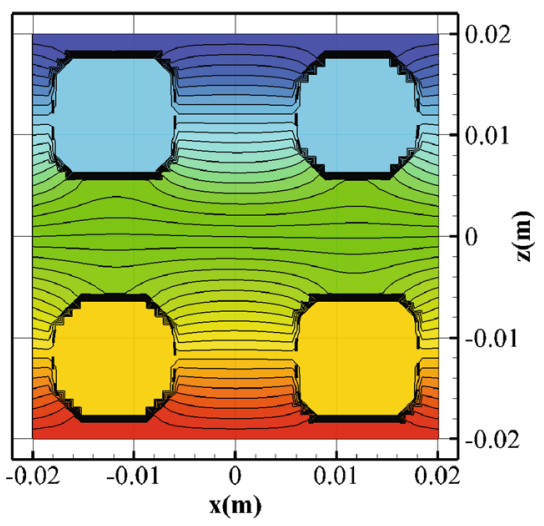

Fig. 18 Distribution of the temperature field in the RVE for configuration 2 and with $k^{(0)}=10^{-6} \mathrm{~W} \mathrm{~m}^{-1} \mathrm{~K}^{-1}$. a $y=-0.012 \mathrm{~m}$. b $y=0 \mathrm{~m}$. c $y=0.012 \mathrm{~m}$

(a)

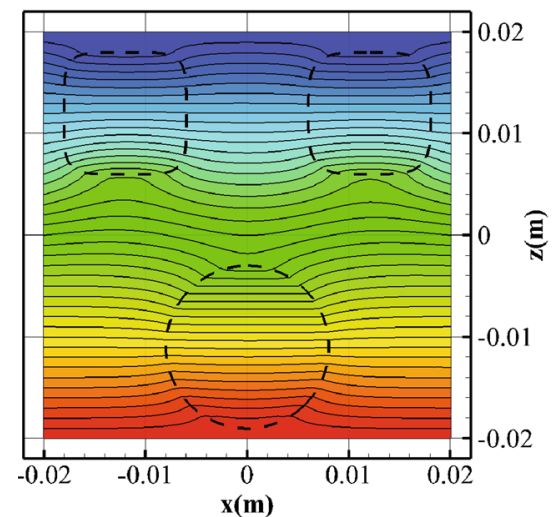

(b)

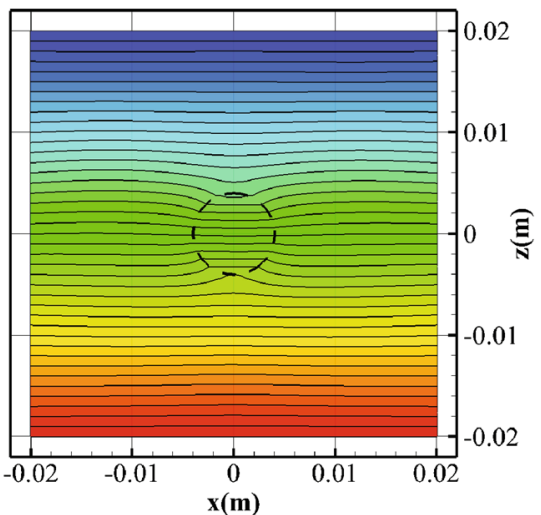

(c)

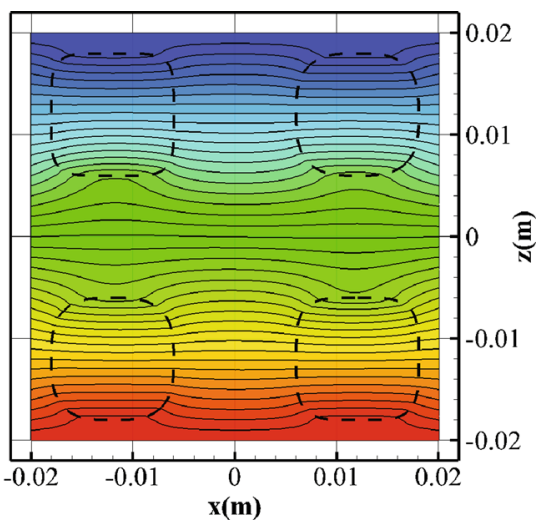

Fig. 19 Distribution of the temperature field in the RVE for configuration 2 and with $k^{(0)}=5 \mathrm{~W} \mathrm{~m}^{-1} \mathrm{~K}^{-1}$. a $y=-0.012 \mathrm{~m}$. b $y=0 \mathrm{~m}$. c $y=0.012 \mathrm{~m}$

(a)

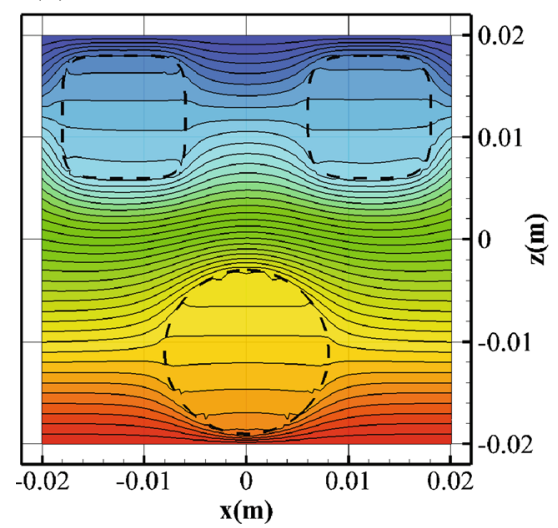

(b)

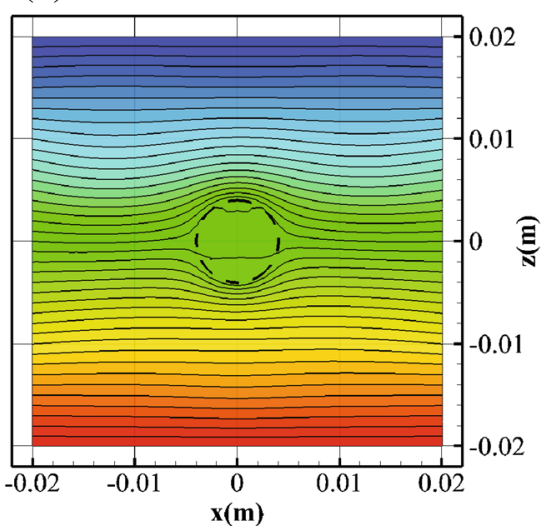

(c)

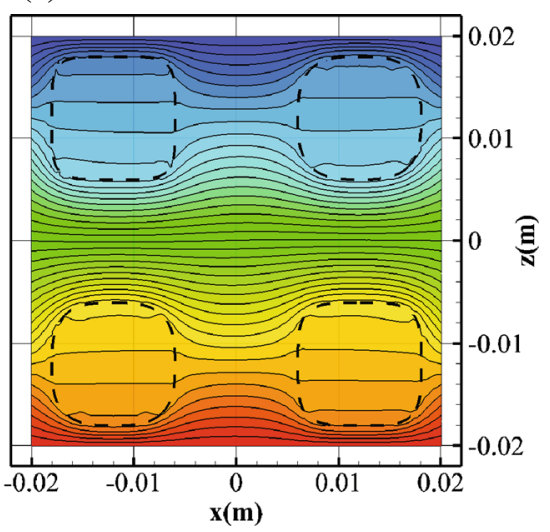

Fig. 20 Distribution of the temperature field in the RVE for configuration 2 and with $k^{(0)}=10^{6} \mathrm{~W} \mathrm{~m}^{-1} \mathrm{~K}^{-1}$. a $y=-0.012 \mathrm{~m}$. b $y=0 \mathrm{~m}$. c $y=0.012 \mathrm{~m}$ 
two-dimensional thermal conduction, the results obtained in the present work can also be useful for dealing with effects of imperfect interfaces in anti-plane elasticity.

Acknowledgments This work is partially supported by the National Natural Science Foundation of China (Grant Number 11202173), the Fundamental Research Funds for the Central Universities (Grant Number SWJTU11CX027) and the Postdoctoral Science Foundation of China (Grant Number 2013M530406).

\section{References}

1. Kapitza PL (1941) Heat transfer and superfluidity of helium II. Phys Rev 60:354-355. doi:10.1103/PhysRev.60.354

2. Johnson R, Little W (1963) Experiments on the Kapitza resistance. Phys Rev 130:596-604. doi:10.1103/PhysRev.130.596

3. Pollack GL (1969) Kapitza resistance. Rev Mod Phys 41:48-81. doi:10.1103/RevModPhys.41.48

4. Nan CW, Birringer R (1998) Determining the Kapitza resistance and the thermal conductivity of polycrystals: a simple model. Phys Rev B 57:8264-8268. doi:10.1103/PhysRevB.57.8264

5. Yvonnet J, He QC, Zhu QZ, Shao JF (2011) A general and efficient computational procedure for modelling the Kapitza thermal resistance based on XFEM. Comput Mater Sci 50:1220-1224. doi:10. 1016/j.commatsci.2010.02.040

6. Le-Quang H, He QC (2011) Eshelby's tensor fields and effective conductivity of composites made of anisotropic phases with Kapitza's interface thermal resistance. Philos Mag 91:3358-3392

7. Javili A, McBride A, Steinmann P (2012) Numerical modelling of thermalmechanical solids with mechanically energetic (generalised) Kapitza interfaces. Comput Mater Sci 65:542-551. doi:10. 1016/j.commatsci.2012.06.006

8. Cheng H, Torquato S (1997) Effective conductivity of dispersions of spheres with a superconducting interface. Proc R Soc Lond Ser A 453:1331-1344. doi:10.1098/rspa1997.0009 1997.0009

9. Lipton R (1997) Variational methods, bounds and size effects for composites with highly conducting interface. J Mech Phys Solids 45:361-384. doi:10.1016/S0022-5096(96)00097-X

10. Lipton R (1997) Reciprocal relations, bounds, and size effects for composites with highly conducting interface. SIAM J Appl Math 57:342-363. doi:10.1137/S0036139995291180

11. Duan HL, Karihaloo BL (2007) Effective thermal conductivities of heterogeneous media containing multiple imperfectly bonded inclusions. Phys Rev B 75:064206. doi:10.1103/PhysRevB.75. 064206

12. Yvonnet J, He QC, Toulemonde C (2008) Numerical modelling of the effective conductivities of composites with arbitrarily shaped inclusions and highly conducting interface. Compos Sci Technol 68:2818-2825. doi:10.1016/j.compscitech.2008.06.008

13. Le-Quang H, Bonnet G, He QC (2010) Size-dependent Eshelby tensor fields and effective conductivity of composites made of anisotropic phases with highly conducting imperfect interfaces. Phys Rev B 81:064203. doi:10.1103/PhysRevB.81.064203

14. Javili A, McBride A, Steinmann P (2013) Numerical modelling of thermomechanical solids with highly conductive energetic interfaces. Int J Numer Method Eng 93:551-574. doi:10.1002/nme. 4402

15. Belytschko T, Black T (1999) Elastic crack growth in finite elements with minimal remeshing. Int J Numer Method Eng 45:601620. doi:10.1002/(SICI)1097-0207(19990620)

16. Moës N, Dolbow J, Belytschko T (1999) A finite element method for crack growth without remeshing. Int J Numer Method Eng 46:131-150. doi:10.1002/(SICI)1097-0207(19990910)
17. Belytschko T, Moës N, Usui S, Parimi C (2001) Arbitrary discontinuities in finite elements. Int J Numer Method Eng 50:993-1013. doi:10.1002/1097-0207(20010210)

18. Ji H, Dolbow JE (2004) On strategies for enforcing interfacial constraints and evaluating jump conditions with the extended finite element method. Int J Numer Method Eng 61:2508-2535. doi:10. 1002/nme. 1167

19. Benvenuti E (2008) A regularized XFEM framework for embedded cohensive interfaces. Comp Method Appl Mech Eng 197:43674378. doi:10.1016/j.cma.2008.05.012

20. Yvonnet J, Le-Quang H, He QC (2008) An XFEM/level set approach to modelling surface/interface effects and to computing the size-dependent effective properties of nanocomposites. Comput Mech 42:119-131. doi:10.1007/s00466-008-0241-y

21. Nistor I, Guiton MLE, Massin P, Moës N, Gé niaut S (2009) An $\mathrm{X}$-FEM approach for large sliding contact along discontinuities. Int J Numer Method Eng 78:1407-1435. doi:10.1002/nme.2532

22. Cheng KW, Fries TP (2010) Higher-order XFEM for curved strong and weak discontinuities. Int J Numer Method Eng 82:564-590. doi: $10.1002 /$ nme. 2768

23. Farsad M, Vernerey FJ, Park HS (2010) An extended finite element/level set method to study surface effects on the mechanical behavior and properties of nanomaterials. Int J Numer Method Eng 84:1466-1489. doi:10.1002/nme.2946

24. Fries TP, Belytschko T (2010) The extended/generalized finite element method: an overview of the method and its applications. Int J Numer Method Eng 84:253-304. doi:10.1002/nme.2914

25. Zhu QZ, Gu ST, Yvonnet J, Shao JF, He QC (2011) Threedimensional numerical modelling by XFEM of spring-layer imperfect curved interfaces with applications to linearly elastic composite materials. Int J Numer Method Eng 88:307-328. doi:10.1002/ nme. 3175

26. Sussmann C, Givoli D, Benveniste Y (2011) Combined asymptotic finite-element modelling of thin layers for scalar elliptic problems. Comput Method Appl Mech Eng 200:3255-3269. doi:10.1016/j. cma.2011.08.001

27. Sanchez-Palencia E (1970) Comportement limite d'un probl ème de transmission à travers une plaque faiblement conductrice. C R Acad Sci Ser A 270:1026-1028

28. Pham HP, Sanchez-Palencia E (1974) Phénomènes de transmission a travers des couches minces de conductivité élevé e. J Math Anal Appl 47:284-309

29. Hashin $Z$ (2001) Thin interphase/imperfect interface in conduction. J Appl Phys 89:2261-2267. doi:10.1063/1.1337936

30. Benveniste Y (2006) A general interface model for a threedimensional curved thin anisotropic interphase between two anisotropic media. J Mech Phys Solids 54:708-734. doi:10.1016/ j.jmps.2005.10.009

31. Gu ST, He QC (2011) Interfacial discontinuity relations for coupled multifield phenomena and their application to the modeling of thin interphases as imperfect interfaces. J Mech Phys Solids 59:14131426. doi:10.1016/j.jmps.2011.04.004

32. Gu ST, Yang D, He QC (2013) Modelling of thermal imperfect interfaces: beyond Kapitza resistance and highly conducting models. (submitted)

33. Dolbow J, Harari I (2009) An efficient finite element method for embedded interface problems. Int J Numer Method Eng 78:229_ 252. doi: $10.1002 / \mathrm{nme} .2486$

34. Harari I, Dolbow J (2010) Analysis of an efficient finite element method for embedded interface problems. Comput Mech 46:205211. doi:10.1007/s00466-009-0457-5

35. Gu ST, Monteiro E, He QC (2011) Coordinate-free derivation and weak formulation of a general imperfect interface model for thermal conduction in composites. Compos Sci Technol 71:12091216. doi:10.1016/j.compscitech.2011.04.001 
36. Gurtin ME, Murdoch AI (1975) A continuum theory of elastic material surfaces. Arch Ration Mech Anal 57:291-323. doi:10. 1007/BF00261375

37. Belytchko T, Parimi C, Moës N, Sukumar N, Usui S (2003) Structured extended finite element method for solids defined by implicit surfaces. Int J Numer Method Eng 56:609-635. doi:10.1002/nme. 686
38. Chessa J, Wang HW, Belytschko T (2003) On the construction of blending elements for local partition of unity enriched finite elements. Int J Numer Method Eng 57:1015-1038. doi:10.1002/ nme.777

39. Fries TP (2008) A corrected XFEM approximation without problems in blending elements. Int J Numer Method Eng 75:503-532. doi:10.1002/nme.2259 